Isaac Boxx, Michael Stöhr, Campbell Carter*, Wolfgang Meier, Temporally resolved planar measurements of transient phenomena in a partially pre-mixed swirl flame in a gas turbine model combustor, Combust. Flame 157 (2010) 1510-1525

* Wright Patterson AFB

The original publication is available at www.elsevier.com

http://dx.doi.org/10.1016/j.combustflame.2009.12.015 
Combustion and Flame

Accepted: Dec 16, 2009

\title{
Temporally resolved planar measurements of transient phenomena in a partially pre-mixed swirl flame in a gas turbine model combustor
}

\author{
I. Boxx ${ }^{1}$, M. Stöhr ${ }^{1}$, C. Carter ${ }^{2}$, W. Meier ${ }^{1}$ \\ 1: Institut für Verbrennungstechnik, Deutsches Zentrum für Luft-und Raumfahrt (DLR), \\ Pfaffenwaldring 38-40, D-70569 Stuttgart, Germany \\ 2: Air Force Research Laboratory (AFRL) / PRAS \\ 1950 Fifth St, Wright-Patterson AFB, OH USA
}

Corresponding Author: Isaac Boxx, Pfaffenwaldring 38-40, D-70569 Stuttgart, Germany, Fax: (+49) 7116862 578, e-mail: Isaac.Boxx@dlr.de 


\begin{abstract}
This paper presents observations and analysis of the time-dependent behaviour of a $10 \mathrm{~kW}$ partially pre-mixed, swirl stabilized methane-air flame exhibiting self-excited thermo-acoustic oscillations. This analysis is based on a series of measurements wherein particle image velocimetry (PIV) and planar laser-induced fluorescence (PLIF) of the $\mathrm{OH}$ radical were performed simultaneously at $5 \mathrm{kHz}$ repetition rate over durations of $0.8 \mathrm{~s}$. Chemiluminescence imaging of the $\mathrm{OH}^{*}$ radical was performed separately, also at $5 \mathrm{kHz}$ over $0.8 \mathrm{~s}$ acquisition runs. These measurements were of sufficient sampling frequency and duration to extract usable spatial and temporal frequency information on the medium to large-scale flow-field and heat-release characteristics of the flame. This analysis is used to more fully characterize the interaction between the self-excited thermo-acoustic oscillations and the dominant flowfield structure of this flame, a precessing vortex core (PVC) present in the inner recirculation zone. Interpretation of individual measurement sequences yielded insight into various physical phenomena and the underlying mechanisms driving flame dynamics. It is observed for this flame that location of the reaction zone tracks large-scale fluctuations in axial velocity and also conforms to the passage of large-scale vortical structures through the flow-field. Local extinction of the reaction zone in regions of persistently high principal compressive strain is observed. Such extinctions, however, are seen to be self healing and thus do not induce blow-out. Indications of auto-ignition in regions of unburned gas near the exit are also observed. Probable auto-ignition events are frequently observed coincident with the centers of large-scale vortical structures, suggesting the phenomenon is linked to the enhanced mixing and longer residence times associated with fluid at the core of the PVC as it moves through the flame.
\end{abstract}




\section{Introduction}

In practical combustors, such as industrial furnaces or gas turbine (GT) combustors, swirling flows are often used because they enable a high energy conversion in a small volume and exhibit good ignition and stabilization behavior over a wide operating range [Gupta et al. (1984); Syred, et al., (1971); Weber and Dugue (1992)]. Beyond a critical swirl number, vortex breakdown occurs [Gupta et al., (1984); Lucca-Negro and O'Doherty, (2001)] leading to the establishment of an inner recirculation zone (IRZ) which transports hot combustion products and radicals back to the flame base. The mixing of the hot products with the incoming fuel-air mixture speeds up and stabilizes the combustion process, enabling operation over a significantly larger range of fuel/air ratios than may otherwise be possible. Swirling flows and flames, however, are also extremely complex and susceptible to various instabilities. On a short time scale the IRZ generated during vortex breakdown does not resemble a solid body vortex at all but is instead composed of many smaller vortices that appear and disappear rapidly [Ji and Gore, (2002); Stopper, et al. (2008); Stöhr, et al. (2009)]. Such vortices can arise from the Kelvin-Helmholz instability in the shear-layer and centrifugal instability induced by the swirl [Wang, et al. (2005)], as well as from random turbulent fluctuations. Energetic large-scale coherent flow structures also play a key role in the turbulence-chemistry interaction of such flames. Of particular interest in recent years is the precessing vortex core (PVC), a three-dimensional unsteady and asymmetric coherent flow structure frequently observed in swirl-stabilized combustors [Froud et al. (1995); Anacleto et al. (2003); Wegner (2004); Syred, (2006); Schildmacher et al., (2006); Fernandes et al., (2006); Valera-Medina et al. (2009)]. This structure tends to appear at the boundary of the IRZ and the shear-layer, strongly influencing local flame dynamics [Syred, (2006)].

In the field of GT combustion for power generation, a major goal is a significant reduction of NOx emissions. This has led in recent years to the concept of lean premixed and 
partially premixed combustion where peak temperatures at near-stoichiometric conditions are avoided [Correa, (1998); Lefebvre, (1999)]. Further, this concept allows the achievement of a quite homogeneous temperature distribution at the turbine inlet and thus reduced thermal load. Unfortunately, lean premixed GT flames are susceptible to thermo-acoustic instabilities driven by the combustion process and sustained by a resonant feedback mechanism coupling pressure and heat release [Keller, (1995); Fernandes, et al. (1996); Paschereit, (1998); Lefebvre (1999); Candel, (2002); Lee and Santavicca, (2003); Syred, (2006), Correa, (1998); Candel, (2002); Lieuwen and Yang, (2005)]. Such oscillations impact the performance, emissions and even structural integrity of GT combustors, and as such, the study of their fundamental behavior and dynamics remains an area of intense interest. The physical and chemical mechanisms driving the instabilities are based on a complex interaction between combustor geometry, pressure, flow-field, mixing, chemical reactions, and heat release. While active and passive control mechanisms have been developed to reduce or even eliminate instabilities in some industrial burners [Syred, (2006); Docquier and Candel, (2002); Emiris and Whitelaw, (2003); Richards, et al. (2003), Mongia, et al. (2003)], the problem is not yet fully understood at a fundamental level.

Another important aspect of GT combustion that is often related to instabilities is the stabilization mechanism of swirled premixed flames. While it is generally known that the mixing of hot products from the IRZ into the fresh gas plays an important role, the contribution from coherent structures such as the PVC (if present) is not well known. Also, it is not clear to what extent autoignition of mixtures of exhaust and fresh gas at elevated temperatures occurs or whether flame propagation by (partially) premixed flamelets is the dominant burning mechanism [Sadanandan, et al. (2008); Guttenfelder, et al., (2006)]. Previous investigations have shown that local flame extinction occurs frequently in GT-like flames [Meier, et al.,(2006)]; however, the details of the interaction between a vortex and a 
flame front have, so far, only been demonstrated for simpler flame configurations [Vagelopoulos and Frank, (2006); Meyer, et al. (2004); Hult, et al. (2005)] and not in GT flames.

The complexity of the combustion process in lean, swirl-stabilized GT flames has led in recent years to a trend toward the application of multiple, complementary measuring techniques, in particular 2D laser measuring techniques. The simultaneous application of particle image velocimetry (PIV) and planar laser-induced fluorescence (PLIF) have, for example, contributed to a better understanding of the flow-field/flame interaction in swirl flames [Petersson, et al. (2007); Tanahashi, et al. (2005); Sadanandan et al. (2008); Stöhr et al. (2009)]. These techniques also provide minimally-intrusive diagnostics in otherwise inaccessible environments such as high-pressure test rigs and combustion chambers. Research groups have performed laser-based measurements in high-pressure test rigs with optical access in order to investigate GT combustion processes under realistic conditions, i.e. preheated air, high thermal loads, high turbulence levels and relevant combustor geometries [Lee, et al. (2000); Meier, et al. (2000); Löfström, et al. (2000); Gord, et al. (2002); Kojima and Nguyen, (2004); Willert, et al. (2006), Griebel, et al. (2007); Janus, et al. (2008); Stopper, et al. (2008); Strakey, et al. (2008), Ax, et al. (2009)]. While these investigations have contributed significantly to a better understanding of certain aspects of GT combustion under pressure, such complex and expensive experiments do not (and, indeed, cannot) result in systematic or comprehensive characterization of swirl flames and are often affected by uncertainties in the boundary conditions, originating, e.g., from limited optical access or a contribution from cooling air to the combustion. Alternatively, the use of model combustors that can be operated under laboratory conditions has proven a promising concept to gain insight into the interplay between the turbulent swirling flow-field and the flame chemistry and to acquire reliable experimental data suitable for model validation. 
The use of optically accessible, laboratory-scale model combustors has been particularly fruitful in the study of thermo-acoustic pulsation in lean swirl-stabilized flames. By combining multiple high-resolution laser measurement techniques with acoustic signal acquisition in a well-controlled system, one may extract phase-resolved velocity and scalar field data over the dominant acoustic pulsation cycle and thus better understand its underlying physical mechanism. Venkataraman et al. [1999] applied phase-resolved imaging of $\mathrm{CH}$ chemiluminescence to study flame-vortex interaction and flame surface area changes over the thermo-acoustic cycle of a lean premixed dump combustor and found that swirl fundamentally alters the combustion instability. Ayoola et al. [2006] demonstrated the viability of using simultaneous $\mathrm{OH}$ and $\mathrm{CH}_{2} \mathrm{O}$ PLIF to determine the phase-dependent local heat-release rate in turbulent, premixed swirl flames. Using this technique (in addition to phase-resolved imaging of $\mathrm{OH}^{*}$ and $\mathrm{CH}^{*}$ chemiluminescence and burst-mode highspeed $\mathrm{OH}$ PLIF) Balachandran et al. [2005] studied flame surface density and local heat release rate of acoustically forced, bluff-body stabilized flames. They observed the flame to wrap around propagating shear-layer vortices, resulting in a transient increase in local flame-surface area and heat-release rate. This phenomenon was further explored by Bellows et al. [2007], using phase-resolved OH-PLIF. In addition to the flame / shear-layer vortex interaction, they also observed unsteady flame lift-off during the phase of the acoustic cycle corresponding to peak axial-velocity and a corresponding decrease in flame-surface area. Subsequent phase-resolved OH-PLIF imaging in the same burner lead Thumuluru and Liewen [2009] to conclude that the flame response is not controlled by any single process, but rather several competing fluidmechanical phenomena.

The present work focuses on the DLR Dual-Swirl Gas Turbine Model Combustor (GTMC). The GTMC is a research-scale swirl burner developed for the study of thermo- 
acoustic oscillations and other aspects of the turbulence-chemistry interaction under technically relevant operating conditions. It consists of a nozzle with two concentric air swirlers fed from a common plenum. The two co-swirling airstreams are separated by an annular ring nozzle supplying liquid or gaseous fuel. The nozzle is mounted in a combustion chamber with almost unrestricted optical access in the visible and ultraviolet regions of the spectrum. The excellent optical access, compact size and moderate thermal load $(7-35 \mathrm{~kW})$ make this burner ideally suited to both point- and planar-laser measurement techniques. As such, it has been the subject of extensive measurement and characterization studies that have relied extensively on the application of various non-intrusive laser-based measurement techniques.

Giezendanner et al. [2003, 2005a] studied a lean $(\varphi=0.75), 10 \mathrm{~kW}$ methane-air flame in this burner and observed a strong, self-excited combustion oscillation at a frequency of approximately $290 \mathrm{~Hz}$. Using planar laser-induced fluorescence (PLIF) of the $\mathrm{OH}, \mathrm{CH}$ and $\mathrm{H}_{2} \mathrm{CO}$ combustion intermediates, laser Doppler anemometry (LDA) and $\mathrm{OH}^{*}$ chemiluminescence imaging, all phase-locked to the dominant oscillation frequency, they determined flame zone location and the cyclic variability of parameters in this burner. Furthermore, they found a strong correlation between heat-release rate and axial velocity near the nozzle exit. Duan et al. [2005] studied the same (10kW) flame using phase-resolved LDA, Raman scattering and PLIF of $\mathrm{OH}$ and $\mathrm{CH}$ and measured pronounced (+/- 33\%) variations in fuel and air flow-rates over the thermo-acoustic cycle and significant deviations from equilibrium composition and temperature near the nozzle. Using two-line OH-PLIF thermometry, Giezendanner et al. [2005b, 2005c] showed these phase-dependent temperature variations were most prominent in the IRZ and that the temperature maximum corresponded to the phase of strongest recirculation. Weigand et al. [2005] identified the basic feedback mechanism behind the dominant $290 \mathrm{~Hz}$ oscillation in this flame. Using phase-resolved LDA, 
PLIF, Raman scattering and $\mathrm{OH}^{*}$ cheminluminescence imaging, they showed that a phasedependent mixing rate of hot products from the recirculation zones with the fresh gas influenced the heat release rate which, in turn, affected thermal expansion and thus had a strong influence on the flow-field, leading to induced periodic motions of the inner and outer recirculation zones.

With an understanding of the underlying mechanism for the thermo-acoustic oscillation in place, research efforts have turned to understanding the physics of the turbulence-chemistry interaction behind this driving mechanism. Weigand et al. [2006] studied three flames in the GTMC: one which burned stably, one oscillating and one close to blowout. Using PLIF of $\mathrm{OH}$ and $\mathrm{CH}, \mathrm{LDA}$ and Raman scattering, they found the flames were not attached at the fuel nozzle (i.e. were partially premixed) and that despite having quite similar mean flow-fields, each displayed significantly different combustion characteristics. They determined the near-field region was dominated by fast mixing and finite-rate chemistry effects and that combustion occurred in thin $(\approx 0.5 \mathrm{~mm})$, strongly corrugated reaction zones. Meier et al. [2006] studied the same three flames using Raman scattering to characterize the mean temperature, major species concentration and mixture fraction throughout each flame. They found wide variation in local, instantaneous mixture fraction and temperature in the near-field and determined that fast mixing near the nozzle exit was accompanied by strong turbulence-chemistry interactions in the form of local extinction and ignition delay. In all three cases, burnout was complete by $50 \mathrm{~mm}$ downstream of the nozzle. Meier et al. [2005] studied flames in the GTMC with a global equivalence ratio of 0.75 and Reynolds numbers ranging from $7500-60,000$ to characterize the influence of different degrees of turbulence on the thermo-chemical state and flame structure. They determined the flames in this burner exhibit more characteristics of diffusion flames than of a uniformly premixed flame. 
Stöhr and Meier [2006] performed a statistical analysis of particle image velocimetry (PIV) measurements using a proper orthogonal decomposition (POD) to show the flow-field of the GTMC is dominated by large-scale, non-stationary, coherent vortical structures. They showed that chief among these is a precessing helical vortex, which occurs in the region between the IRZ and the incoming gas. Subsequently, Stöhr and Meier [2007] used phaseresolved PIV to show the principal motion of the PVC about the vertical axis does not correlate with the thermo-acoustic oscillation. However, using simultaneous, phase-resolved PLIF and PIV, Stöhr et al. [2009] observed that the PVC undergoes significant structural change during the thermo-acoustic oscillation cycle, particularly in the transverse plane. Taking advantage of the fact that $\mathrm{OH}$ is present in super-equilibrium concentration in the reaction zone, Sadanandan et al. [2008] showed that strong gradients in the OH-PLIF signal serve as a useful marker of the reaction zone location in these flames. Applying this principle, they used simultaneous OH-PLIF and PIV in the $10 \mathrm{~kW}$ flame to investigate flow-field / reaction zone interactions. They deduced that small vortices in the shear layers at the edge of the recirculation zones play an important role in flame stabilization.

Previous research, with its extensive reliance upon stochastic and phase-resolved measurement techniques, has generated extensive insight into the flow-field, thermochemical, thermo-acoustic and operational characteristics of this research burner. An extensive database of high resolution experimental measurements suitable for numerical and code-validation studies has been acquired. Numerical simulations of the flame under conditions identical to those measured are currently underway [Widenhorn, (2009)]. Thorough analysis, in particular using spectral and POD methods, has reinforced insight gained by these techniques. A complete fundamental understanding of the turbulencechemistry interaction in this flame, however, remains elusive. This is because even the best phase-averaged measurements only isolate mean and statistical cyclic variations of thermodynamic and thermo-chemical properties. Information on temporally developing, transient or Boxx et al., Combust. Flame (2010). Accepted for Publication Dec. 16, 2009. 
non-periodic combustion processes is lost. RANS and URANS simulations are similarly limited. A fully resolved (temporally and spatially) direct numerical simulation with detailed chemistry and sufficient run duration to be statistically relevant is well beyond the state of the art and will likely remain so well into the future.

A logical extension of the phase-resolved measurements and quasi-steady numerical simulations described above is the acquisition of long-duration, time-resolved measurements of similar flow-field quantities. As demonstrated by Upatnieks et al. [2004], Steinberg et al. [2008] and Boxx et al. [2009a, 2009b, 2009c], such measurements have the clear benefit of allowing one to track specific events in the flow-field and reaction zones as they occur, greatly simplifying the interpretation of such data. Acquisition of a statistically significant number of cycles (e.g., several 100) per run also renders such interpretations more reliable. Long-duration, temporally resolved measurements also enable one to capture temporally unpredictable phenomena such as flashback [Konle et al., (2008); Konle and Sattelmayer (2009)], turbulent flame extinction [Böhm et al. (2009)] and misfire events in internal combustion engines [Fajardo and Sick, (2009)]. The ability to acquire these measurements over several hundred consecutive cycles also allows a short (absolute) acquisition time.

The aim of this paper is to present results from a recent experimental study, wherein we applied simultaneous 3-component stereoscopic PIV and OH-PLIF at a $5 \mathrm{kHz}$ repetition rate over long periods ( $>0.8 \mathrm{~s}$ per run) to study the turbulence-chemistry interaction in a partially premixed, swirl-stabilized turbulent flame generated by the DLR Gas Turbine Model Combustor. This data is used to identify quasi-instantaneous/phenomenological behavior of the flame in the IRZ and inner shear-layer, as well as to extract temporal- and spatialfrequency information on the turbulence-chemistry interaction therein. Characteristic phenomena related to the $\mathrm{PVC} /$ reaction-zone interaction, thermo-acoustic oscillation and general combustion behavior are identified and discussed. 


\section{Burner and Flames}

As the GTMC has been extensively characterized and there exist numerous detailed descriptions of it in the literature, only a short description is presented here. Figure 1 shows a schematic of the GTMC. It consists of two parts, the combustion chamber and the plenum with the gas film nozzle. The combustion chamber has a square cross-section measuring 85 $\mathrm{mm} \times 85 \mathrm{~mm}$ and stands $110 \mathrm{~mm}$ tall. The walls of the chamber are quartz plates held at the corners with Inconel ${ }^{\circledR}$ alloy posts (dia. $10 \mathrm{~mm}$ ). The chamber is capped with a steel plate with a central exhaust tube (dia. $40 \mathrm{~mm}$, length $50 \mathrm{~mm}$ ) over a conical contraction. Dry, room temperature air enters the combustion chamber through a central nozzle (diameter $15 \mathrm{~mm}$ ) and a concentric annular nozzle (i.d. $17 \mathrm{~mm}$, o.d. $25 \mathrm{~mm}$ contoured to an o.d. of $40 \mathrm{~mm}$ ). The air is co-swirled using two sets of swirl vanes beneath the nozzles. The swirlers are fed from a common plenum. Non-swirling $\mathrm{CH}_{4}$ enters the chamber via a ring of 72 channels $(0.5 \mathrm{~mm} \mathrm{x}$ $0.5 \mathrm{~mm}$ ) located between the two air nozzles. The exit planes of the fuel and central air nozzle stand $4.5 \mathrm{~mm}$ below the exit plane of the outer air nozzle.

The burner is equipped with multiple ports for pressure transducers and microphones. In the present study, only two of the ports were used: one mounted in a corner post of the combustion chamber at $y=16 \mathrm{~mm}$ and the other in the wall of the air plenum. The signals of each microphone (Brüel \& Kjaer, Type 4939) were sampled at $20 \mathrm{kHz}$ via a multichannel A/D converter, which also (simultaneously) sampled and logged the trigger signal to the intensifier used with the PLIF detection camera.

The flame studied in this work corresponds to that described as "Flame B' in the work of Weigand et al. [2006]. Fuel and air were metered separately into the burner using electromechanical mass flow controllers at rates of 12.3 and $281 \mathrm{~g} / \mathrm{min}$, respectively. This corresponds to a flame of $10 \mathrm{~kW}$ thermal power output, an equivalence ratio of 0.75 and swirl number of 0.55 . Approximately $4 \%$ of the swirled co-flow air was delivered to the burner 
through a fluidized bed particle seeder. Flow-rates of the fuel and seed and co-flow air were monitored throughout the experiment via calibration-standard Coriolis mass flowmeters (Siemens Sitrans-FC MassFlo 2100, Model DI-1.5, DI-3 and DI-15 respectively).

The burner was operated at $10 \mathrm{~kW}$ for at least twenty minutes prior to each experiment run to allow it to heat up and thermally stabilize. During this warm-up period, no seed particles were delivered to the air flow. Shortly before a measurement, the bulk air flow was reduced by approximately $4 \%$ and the same amount was metered into the flow via the particle seeder using a separate electromechanical flow controller. This ensured reliable, reproducible seed density for each run, while minimizing seed-buildup on the combustor windows during the warm-up period.

\section{Measurement Technique}

The combined stereo-PIV/OH-PLIF measuring system developed for this work has been described previously [Boxx, et al. (2009a, 2009c)]. As such, only a brief description is provided here.

\section{$\underline{\text { Planar Laser-Induced Fluorescence }}$}

The OH-PLIF system consists of a conventional (albeit modified) frequency-doubled dye laser and an intensified CMOS camera. The dye laser (Sirah Cobra-Stretch HRR, using Rhodamine 6G in ethanol) was pumped with a frequency-doubled, Q-switched, diodepumped solid state Nd:YLF laser (Edgewave IS-811E). At $5 \mathrm{kHz}$, the pump laser delivered $3.8 \mathrm{~mJ} /$ pulse (19 W average output) at $523 \mathrm{~nm}$ and had an $8.5 \mathrm{~ns}$ pulse duration. At $5 \mathrm{kHz}$, the time-average output from the dye laser at $283.2 \mathrm{~nm}$ was $\approx 0.5 \mathrm{~W}$ or $100 \mu \mathrm{J} / \mathrm{pulse}$. The laser line width at $566 \mathrm{~nm}$ was $0.06 \mathrm{~cm}^{-1}$. Wavelength tuning of the dye laser to the peak of the isolated $\mathrm{Q}_{1}(7)$ line of the $\mathrm{A}-\mathrm{X}$ (1-0) transition of $\mathrm{OH}$ was checked daily using a setup consisting of a laminar reference flame and a photomultiplier tube for fluorescence detection. 
The $283.2 \mathrm{~nm}$ beam was formed into a sheet of $\approx 40 \mathrm{~mm}$ (high in the probe region) using two fused silica lenses $\left(f_{\text {plif1 }}=-25 \mathrm{~mm}, f_{\text {plif2 }}=250 \mathrm{~mm}\right)$ in a cylindrical telescope configuration and focused to a waist using a third cylindrical lens $\left(f_{\text {plif3 }}=500 \mathrm{~mm}\right)$. The $283 \mathrm{~nm}$ laser sheet was overlapped with the PIV laser sheet using a pair of dichroic mirrors (see Fig. 1). The PIV and PLIF sheets were overlapped in the near and far fields and also in the probe region; this process was checked daily, and the overlap was found to be consistently very good. Sheet thicknesses were measured by translating a narrow slit through each beam and using a photodiode to record the spatial distribution; with this approach the $283 \mathrm{~nm}$ beam was determined to be $\approx 400 \mu \mathrm{m}$ at the probe volume.

Fluorescence signal was acquired with a CMOS camera (LaVision HSS6), external twostage, lens-coupled intensifier (LaVision HS-IRO) and a fast Cerco 45mm, f/1.8 lens that is AR coated for the UV. Background luminosity was reduced using a $500 \mathrm{~ns}$ intensifier gate and elastic (particle) scattering at $283 \mathrm{~nm}$ was blocked using a high-transmission $(>80 \%$ at $310 \mathrm{~nm}$ ) bandpass interference filter (Custom fabrication - Laser Components $\mathrm{GmbH}$ ) and a color glass filter (1 mm thick WG295 Schott glass). A correction/calibration for spatial variation of the laser sheet-intensity and camera/intensifier sensitivity was accomplished using a mean image derived from laser-induced fluorescence of acetone in the quartz-plate duct near the jet-exit. Run-to-run background and camera noise (equivalent to $\approx 1 \%$ of the dynamic range) were corrected using a 1000 frame ensemble average acquired while the lasers were blocked.

\section{$\underline{\text { Particle Image Velocimetry }}$}

The stereoscopic PIV system is based on a dual-cavity, diode-pumped, solid state Nd:YAG laser (Edgewave, IS-611DE) and a pair of CMOS cameras (LaVision HSS5). The laser produces $2.6 \mathrm{~mJ} /$ pulse at $532 \mathrm{~nm}$ at a repetition rate up to $10 \mathrm{kHz}$. As the laser is pulseenergy (as opposed to quasi-cw average power) limited, this corresponds to $13 \mathrm{~W}$ per head at $5 \mathrm{kHz}$. Pulse duration is $\approx 14 \mathrm{~ns}$. Pulse timing separation for the PIV system was $\Delta t=20 \mu \mathrm{s}$, 
with the OH-PLIF excitation pulse temporally interlaced between the first and second PIV pulses of each measurement cycle.

As with the PLIF system, three cylindrical lenses were used to form the sheet: $f_{\text {piv1 }}=-25$ $\mathrm{mm}, f_{\text {piv } 2}=300 \mathrm{~mm}$, and $f_{\text {piv } 3}=1000 \mathrm{~mm}$. To minimize noise arising from inter-frame particle dropout, the beam waist was located somewhat beyond the probe region. The sheet thicknesses were measured in the same manner as for the PLIF beam; at the probe volume, the sheet thicknesses were $\leq 0.7 \mathrm{~mm}$. Mie scattering from titanium dioxide $\left(\mathrm{TiO}_{2}\right)$ particles (nominal diameter $0.5 \mu \mathrm{m}$ ) seeded into the flow was imaged using the pair of CMOS cameras mounted equidistant from opposite sides of the laser sheet (see Fig. 1). Each PIV camera has sufficient on-board memory (2.6 GB) for 4096 dual-frame images (i.e., 0.8 seconds imaging) at $512 \times 512$ pixel resolution. For both PIV cameras, scattered light was collected with a Tokina $100-\mathrm{mm}$ focal length lens with the f-stop set to $\mathrm{f} / 5.6$, and image-blur due to off-axis defocusing was corrected using a Scheimpflug adaptor between the lens and camera. Perspective distortion was corrected using a dual-plane, three dimensional imaging target (LaVision Type 7). The same target was used to map the fields of view of the stereoscopic PIV and PLIF systems to one another. Image mapping, calibration, and particle crosscorrelations were completed using a commercial, multi-pass adaptive window offset crosscorrelation algorithm (LaVision $\mathrm{DaVis} 7.2)$. Final window size and overlap were $16 \times 16$ pixels and $50 \%$, respectively. This corresponds to a spatial resolution of $1 \mathrm{~mm}$ and vector spacing of $0.5 \mathrm{~mm}$. Based on the \pm 0.1 pixel uncertainty of the cross-correlation peak-finding algorithm, the random uncertainty of the PIV measurements is estimated to be $\pm 0.3 \mathrm{~m} / \mathrm{s}$.

\section{$\underline{\text { Results and Discussion }}$}

Figure 2a shows a mean flow-field of the GTMC, acquired using a lower repetitionrate PIV system with a larger field of view. Although acquired with a different measurement system as part of another study, this figure shows the dominant flow-field characteristics of 
the combustor. The flow swirls counter-clockwise about the vertical axis (as viewed from above) and the highest flow velocities are seen in the conically shaped inflow of fresh gases. The velocity values in the measurement plane are indicated by the arrows and the out-of-plane component $(\mathrm{Vz})$ by the color bar. The flow-field is further characterized by a strong IRZ. There also exists a weaker, outer recirculation zone (ORZ), appearing in the lower corner regions of the burner. This zone results from the confinement effect of the combustion chamber and is known to also play a role in flame stabilization. There exist two shear layers; one between the inflow and the IRZ and one between the inflow and the ORZ. With hot combustion products being continuously mixed with the fresh fuel-air mixture in the inner shear-layer (ISL), combustion is stabilized and the flame tends to reside there. However, as will be shown later, combustion does frequently occur beyond the ISL, and the turbulencechemistry interaction is frequently strong enough to force the reaction zone well beyond the mean-shear-layer region. Figure $2 \mathrm{~b}$ shows a mean velocity field acquired with the current system, illustrating the relative field of view and spatial resolution. Also shown in this Fig. $2 \mathrm{~b}$ are the locations of the three monitor points used to determine the power spectra of axial velocity discussed later in the paper.

Figure 3a shows an OH-PLIF image acquired during this study. As noted earlier, the imaging region extends from one side of the combustion chamber to the other, spanning approximately $85 \mathrm{~mm}$. The laser sheet extends from the exit plane to approximately $40 \mathrm{~mm}$ downstream. Mean background flame luminosity has been subtracted and spatial nonuniformity of the laser sheet and imaging system response have also been corrected, as noted above. In this figure, the regions without $\mathrm{OH}$ (dark) represent gas at low-to-medium temperatures $(T<1500 \mathrm{~K})$, e.g. fresh fuel/air mixtures, possibly with some admixture of burned gas. From Raman measurements in the same flame, it is known that there is a significant mixing of exhaust gas with fresh gas near the nozzle and the burned/unburned gas mixture can cover a wide temperature range $(300->1500 \mathrm{~K})$. The light-grey regions (i.e. Boxx et al., Combust. Flame (2010). Accepted for Publication Dec. 16, 2009. 
those with moderate-to-high $\mathrm{OH}$ signal levels) within the IRZ and ORZ consist primarily of gas at high temperature $(>1500 \mathrm{~K})$. The brightest regions of the image are indicative of regions containing super-equilibrium concentrations of $\mathrm{OH}$. Although $\mathrm{OH}$ concentrations decay in the high temperature gas over a significantly longer timescale than that of the reactions in the flame front, the OH-PLIF signal may nonetheless be used to determine reaction zone location. Sadanandan, et al. [2008] showed that, for this flame, the largest magnitude $\mathrm{OH}$-gradients result from super-equilibrium concentrations of $\mathrm{OH}$ and are thus representative of the reaction zone of the flame. Lower magnitude $\mathrm{OH}$-gradients downstream of the contiguous reaction zone tend to be indicative of mixing and diffusion of $\mathrm{OH}$ as it moves away from the reaction zones and from mixing of hot combustion products in the IRZ. Figure $3 b$ shows the PLIF image from Fig. 3a after the application of a Sobel gradient-filter. Consistent with previous studies on this flame, the reaction zone is seen to be highly corrugated, continuous over several centimeters and to reside primarily in the shear-layer between the IRZ and the incoming unburned gas. A lower magnitude gradient is seen at the interface between the ORZ and the unburned gas, probably representing a non-reacting mixture of high- and low-temperature gases.

\section{Power Spectra}

Figure 4 shows the power spectra of the axial component of velocity, measured at three monitor points (see Fig. 2). The monitor points correspond to locations within the ISL (a), the IRZ (b) and the jet (c). The plotted data represent the average power spectra of measurements acquired over seven separate acquisition runs or approximately 5.6 seconds. Also shown in Figure 4 are the power spectra of the chemiluminescence (d) and the acoustic (e) signals. The acoustic data reveal a strong peak centered at $308 \mathrm{~Hz}$, with run-to-run variation in peak location of approximately +/- 4Hz. A strong peak at $308 \mathrm{~Hz}$ is also visible in Boxx et al., Combust. Flame (2010). Accepted for Publication Dec. 16, 2009. 
chemilumenscence, IRZ and jet axial-velocity power spectra. This peak clearly corresponds to the dominant thermo-acoustic pulsation frequency for this flame. We note this frequency differs from the $290-295 \mathrm{~Hz}$ measured in previous studies; however, the absolute difference is only $4 \%$. Furthermore, the fact that the peak appears at the same frequency in the acoustic, velocity and chemiluminescence data indicates measurement error or data aliasing is not the cause of this shift. Rather, the difference is believed to arise from sensitivity of the thermoacoustic pulsation to boundary conditions such as ambient temperature and pressure conditions and to deviations of mass flow rates within measurement uncertainty.

These spectra yield significant insight into the global dynamics of the flame. As noted above, there exists a strong peak in the acoustic, chemiluminescence and IRZ velocity spectra at $308 \mathrm{~Hz}$, confirming the thermo-acoustic pulsation is a dominant parameter in this flame. However, there also exist strong secondary peaks in the velocity spectra at the jet and IRZ locations and a different primary peak altogether in the ISL, indicating the presence of other significant fluid dynamic phenomena. The dominant peak in the power spectrum of axial velocity for the ISL lies at $515 \mathrm{~Hz}$. The power spectrum of axial velocity within the jet and IRZ also shows clear secondary peaks at $515 \mathrm{~Hz}$, further indicating the presence of a strong, periodic flow structure in the flame. The peak (at $515 \mathrm{~Hz}$ ) in the axial velocity spectra is likely the result of the helical, precessing vortex core (PVC) previously identified by Stöhr and Meier [2006, 2007]. A helical PVC is a long, tornado-like coherent vortical structure which corkscrews about the vertical axis of the flame. The presence of such structures in reacting swirl flows was predicted in the numerical work of [Roux et al., (2005)] and confirmed experimentally by Stöhr and Meier [2006, 2007]. The PVC was seen to reside in the ISL between the incoming unburned gases and the IRZ and its presence would explain the strong peak in the axial velocity spectra there.

No equivalent peak is visible in the acoustic or chemiluminescence spectra. As chemiluminescence and thermo-acoustic pulsation are related primarily by time-dependent Boxx et al., Combust. Flame (2010). Accepted for Publication Dec. 16, 2009. 
variations in heat-release, this suggests the PVC is primarily fluid-dynamic in nature and does not directly impact the global heat-release rate of the flame. This is consistent with the finding of Stöhr and Meier [2007], who determined the principal motion of the PVC not to be directly correlated with the thermo-acoustic oscillation. This does not, however, indicate the two act independently. The spectra for both the jet and ISL locations show a small secondary peak at $205-210 \mathrm{~Hz}$. The magnitude of the difference $(305-310 \mathrm{~Hz})$ in peak frequency from that of the PVC indicates a superposition of the effects of both phenomena. It is not clear from this data whether the two are coupled or simply superimposed. Stöhr et al. [2009] observed a (thermo-acoustic-driven) phase-dependent variation in the flow structure in the transverse (xz) plane of the combustor chamber, with the unburned gas varying from an annular to a spiral configuration. Although this was not observed to fundamentally alter the nature of the helical PVC, it may affect the in-plane dynamics of it. Such an effect could explain the secondary peak we see in the axial velocity spectra.

\section{$\underline{\text { POD Analysis }}$}

To better characterize the nature of the fluid-dynamic phenomena responsible for the peaks in the power spectra, a proper orthogonal decomposition (POD) based analysis was performed on the PIV data. POD is a well-established technique in the field of fluid mechanics [Berkooz, et al. (1993)]. The result of the POD is a set of orthogonal eigenmodes, representing coherent flow structures ordered by their contribution to the turbulent kinetic energy. For each eigenmode, temporal mode coefficients can be obtained by taking the scalar products of the instantaneous flow-fields with the eigenmode. In the present case, the POD was calculated using the method of snapshots [Sirovich, (1987)] based on the time-series of 2048 flow-field measurements.

From the temporal mode coefficients of the first three eigenmodes (and the known temporal separation between frames), the corresponding power spectra were determined. 
These spectra are presented in Figure 5. The spectra of eigenmodes 1 and 2 show a strong peak at $514 \mathrm{~Hz}$. Comparing this to the power spectra of axial velocity taken in the ISL, it is clear this peak corresponds to the same characteristic flow structure. Similarly, the spectra also show secondary peaks at 205 and $822 \mathrm{~Hz}$ (or approximately $+/-308 \mathrm{~Hz}$, the thermoacoustic frequency).

Vector plots of the first and second spatial eigenmodes are shown in Figure 6. Consistent with the findings of Stöhr and Meier [2006], these two eigenmodes reveal a series of vortices oriented symmetrically about the central axis. Although the field of view of the PIV measurements is limited to $\approx 30 \mathrm{~mm}$ and is positioned asymmetrically about the centerline, these eigenmodes yield important insight into the global flow-field structure. Examination of the major vortices present in the first eigenmode reveals that both rotate in the same direction; counter-clockwise. The dominant vortices in the second eigenmode also both rotate counter-clockwise. However, the streamlines also indicate a pair of smaller, clockwiserotating vortices at $\mathrm{x}=+/-5 \mathrm{~mm}, \mathrm{y}=0 \mathrm{~mm}$ in the second spatial eigenmode. When the first two eigenmodes are superimposed on the mean flow-field with the corresponding multiplicative (scalar) constants, one sees the alternating "zig-zag" pattern characteristic of the PVC. This finding is consistent with that of Stöhr and Meier [2006], who showed the loworder (i.e., the most energetic) eigenmodes of a set of 200 statistically uncorrelated PIV measurements acquired in a similar flame in the same burner represent a helical PVC. A reconstruction of consecutive flow-field measurements based on just the first two eigenmodes clearly shows the oscillating pattern of vortex appearance and propagation one would expect from a two dimensional (planar) cut through a helical PVC located about the central axis of the nozzle. Thus, the first two eigenmodes clearly represent the PVC and indicate it is the dominant vortical structure in this flow-field.

The power spectrum of the multiplicative constants for the third eigenmode shows a strong peak at $308 \mathrm{~Hz}$, indicating this mode corresponds to the thermo-acoustic oscillation Boxx et al., Combust. Flame (2010). Accepted for Publication Dec. 16, 2009. 
cycle. The vector field representation of the third spatial eigenmode (shown in Fig. 6) shows no clear evidence of vortices. Rather, it appears to show primarily up-down movement along the central (vertical) axis and a spreading near the nozzle, where high-velocity, swirled fresh gas enters the chamber. This suggests the thermoacoustic oscillation acts primarily in an updown or 'piston-like' motion along the central vertical axis but does not result in the formation of coherent vortical structures. This is consistent with the work of Weigand et al. [2006], who showed the thermoacoustic oscillation results in a wider, flatter flame zone compared to cases without significant oscillations.

The POD analysis shows, as expected from previous studies, the dominant flow-field structures in this flame are a helical PVC and a thermo-acoustic oscillation that acts predominantly along the vertical axis. Secondary peaks in the spectra indicate an interaction between the themoacoustic pulsation and the PVC, the nature of which is not immediately clear. The power of applying the POD to time-resolved planar measurements such as these is that it allows one to directly determine spatio-temporal scale, frequency and phase information about specific flow structures and to track their progression through the data sequence.

\section{$\underline{\text { Phenomenological Data }}$}

In addition to extracting frequency and structural information via analysis of time series measurements, inspection of individual measurement sequences yields significant new insight into turbulence-chemistry interaction of the flame. Presented below is a sample measurement sequence acquired over $3 \mathrm{~ms}$ or approximately one thermo-acoustic cycle. It illustrates phenomena ranging from vortex-flame interaction and local flame extinction to burnout of unreacted gas downstream of the reaction zone and probable auto-ignition events upstream of 
it. The simultaneous acquisition of both a reaction zone marker and three component velocity field data enables mutually reinforcing interpretations of each observed phenomenon.

\section{$\underline{O H}$ PLIF}

Figure 8a shows a sequence of 15 consecutive images of OH-PLIF, corresponding to about 1 cycle of the thermo-acoustic oscillation. The images in this sequence were cropped to include only the region of overlap between the OH-PLIF and the PIV measurements. The highest $\mathrm{OH}$ LIF signals are found in the region $y<20 \mathrm{~mm}$, where reactions predominantly take place. The super-equilibrium concentrations of $\mathrm{OH}$ formed in the reaction zones decay towards equilibrium levels over several milliseconds [Sadanandan et al., (2008)]. During this time, at the flow velocities present in the flame, a pocket of gas would convect by $10-20 \mathrm{~mm}$ downstream. The highest OH LIF signal levels can thus be regarded as representative of "young" $\mathrm{OH}$, i.e. $\mathrm{OH}$ which has just been formed in the reaction zone. Thus, further downstream, the $\mathrm{OH}$ concentrations are expected to be close to equilibrium levels. However it should be noted that the local temperature and composition can also influence $\mathrm{OH}$ number density and thus signal intensity.

Even absent the simultaneously acquired velocity field data, this sequence is illustrative of the complexity of the instantaneous $\mathrm{OH}$ field compared to that seen in phaseaveraged measurements. In the first three frames, the reaction zone moves upstream, elongates and moves to the left of centerline, before flattening and moving downstream again by frame 6 . In frames 7 to 10 , the reaction zone again moves upstream, this time extending to the right of centerline. In frame 7, two isolated pockets of $\mathrm{OH}$ appear near the exit plane of the nozzle $(\mathrm{y} \approx 5 \mathrm{~mm}, \mathrm{x} \approx 3-8 \mathrm{~mm})$. These pockets of $\mathrm{OH}$ are not part of the contiguous, inplane $\mathrm{OH}$ field, indicating they either appear from through-plane flame propagation, throughplane bulk transport, or auto-ignition of the fuel-air mixture near the exit. These regions of $\mathrm{OH}$ grow and merge with the contiguous flame front over the next several frames. Another Boxx et al., Combust. Flame (2010). Accepted for Publication Dec. 16, 2009. 
example of an isolated $\mathrm{OH}$ pocket appears in frame 15. In frames 11 and 12, we see a rapid decrease in $\mathrm{OH}$ signal magnitude along the light-dark interface on the left hand side of the image, indicating, presumably, local extinction of the flame. The extinguished region moves downstream and grows larger before beginning to recover near the final frame.

Figure 7 shows the measured pressure signal acquired simultaneously with the PIV / PLIF measurements. It is important to note that the PIV / PLIF measurements were acquired at constant frame-rate simultaneously with the acoustic signal but were not synchronized to it. The points shown along the curve correspond to the measured point along the acoustic cycle specific to each frame in Fig. 8a. With that in mind, Fig. 7 demonstrates that the OH PLIF image sequence shown in Fig. 8a behaves in a manner consistent with what one would expect from the phase-averaged measurements of Giezendanner et al. [2003]. For example, the OHcontaining region reaches its maximum upstream position in frames 2 through 5, which surround the negative maximum on the acoustic cycle. The $\mathrm{OH}$ zone dims and moves axially downstream in frames 6 through 10, corresponding to the rise of the acoustic cycle. Large regions of high $\mathrm{OH}$ concentration are visible downstream and to the right of centerline in frames $11-15$, the region surrounding the positive peak of the acoustic cycle. Qualitatively similar characteristics are apparent in phase-averaged OH LIF measurements of Giezendanner et al. [2003]. Although it is beyond the scope of the present work, Fig. 7 also demonstrates that it is possible to phase-average the OH LIF and PIV measurements in post-processing in order to directly compare them to earlier phase-resolved measurements acquired at much lower frame rates.

\section{$\underline{\text { OH Gradient Field }}$}

Figure $8 \mathrm{~b}$ shows the same sequence after application of a Sobel gradient filter. Consistent with previous studies, the largest magnitude gradients in the $\mathrm{OH}$ field observable in these measurements occur in thin regions along the interface between the incoming unburned gas Boxx et al., Combust. Flame (2010). Accepted for Publication Dec. 16, 2009. 
and the IRZ. Sadanandan, et al. [2008] have shown that for this flame the largest magnitude gradients result from super-equilibrium concentrations of $\mathrm{OH}$ and are thus representative of the reaction zone of the flame. Lower magnitude gradients downstream of the contiguous reaction zone are indicative of mixing and diffusion of $\mathrm{OH}$ as it moves away from the reaction zones and from mixing of hot combustion products in the IRZ.

In the upper left hand side of images 1 through 3 there exists a region without measurable quantities of $\mathrm{OH}$ downstream of the contiguous reaction zone. Although not visible in the displayed images, this region was observed to result from the engulfment of a region of unburned gas near the nozzle during a large fluctuation of the flame sheet. The rapid decay and disappearance of this region indicates either burnout or mixing of unburned gas with combustion products before eventually burning out downstream. The relatively low magnitude gradient in the $\mathrm{OH}$ field compared to those in the contiguous reaction zone upstream suggests burn-out occurs there under unfavorable (e.g. very lean) conditions.

\section{Flame Location}

Figures $8 \mathrm{c}$ and $\mathrm{d}$ show the reaction zone location (based on a threshold outlining the highest magnitude $\mathrm{OH}$-gradients) overlaid on the contours of axial- and through-plane velocity, respectively. The vectors overlaid on top represent the in-plane components of velocity. For clarity, the vector overlay shows only every second computed vector. In Figs. $8 \mathrm{c}$ and $\mathrm{d}$, the red background contours represent strong positive axial- and through-plane velocity, respectively. Green represents low- (i.e. near zero, positive or negative) magnitude velocities and blue represents strongly negative velocities. Prior to display, the velocity field measurements were processed using a 2048-set POD, which was bandpass-filtered before the velocity field was reconstructed. The spectrum was cut off after the first 150 spatial eigenmodes, which corresponded to approximately $70 \%$ of the energy content of the signal, as the lack of discernable spatial or temporal structure and very low energy content in the higher Boxx et al., Combust. Flame (2010). Accepted for Publication Dec. 16, 2009. 
modes indicated primarily noise. This filtering was necessary due to the relatively course velocity resolution of the PIV system $(\approx+/-0.3 \mathrm{~m} / \mathrm{s})$ at low velocity, which (as will be demonstrated later) locally affects derivative quantities such as vorticity and strain. The flame was observed to respond primarily to large-scale fluctuations in the flow and the POD is a very effective scale decomposition for the identification of energetic flow structures [Adrian, et al. (2000)]. No significant modification of the mid- to large-scale structure of the velocity field was observed to result from this filtering.

Figure $8 \mathrm{c}$ shows the reaction zone to reside primarily in regions of low axial velocity, represented by the green regions in the colour contour. Such regions tend to occur along the interface between high axial-velocity incoming fluid (i.e., unburned gas from the nozzle) and the lower- or negative-magnitude axial-velocity fluid in the IRZ. Here the flame is sustained by the well mixed, unburned incoming gas from the nozzle on one side and the hightemperature, combustion-radical laden burned gas transported from downstream. Although transient effects occasionally drive the reaction zone into regions of high axial velocity, it tends not to remain there.

The contour of the reaction zone reaches its maximum upstream position in the low velocity region between the shear-layers that are located on either side of the origin. The flattening and downstream propagation of the reaction zone previously noted in the frames 5 through 7 of the $\mathrm{OH}$ image sequence clearly correlates to a mass of high axial-velocity gas issuing from the nozzle. As this high axial-velocity fluid exits the measurement region, the reaction zone propagates back upstream coincident with a mass of negative axial-velocity fluid, elongating and wrinkling in the process.

Figure $8 \mathrm{~d}$ shows there is little correlation between the instantaneous spatial location of the flame contour and the iso-contours of Vz-velocity, beyond a tendency to also reside in regions of lower velocity magnitude. The relative insensitivity of the flame location to the VzBoxx et al., Combust. Flame (2010). Accepted for Publication Dec. 16, 2009. 
velocity field may be due to lower magnitude fluctuations compared to those of axial velocity, which is strongly affected by the thermo-acoustic oscillation.

\section{$\underline{\text { Flame-Vortex Interaction }}$}

Figure $8 \mathrm{e}$ shows the same measurement sequence overlaid on the instantaneous vorticity field in order to better identify vortex-flame interaction. In frames 1 through 5 , a vortical structure of approximately $8-10 \mathrm{~mm}$ diameter appears to the left of the origin and propagates downstream. In frames 5 through 10, a vortical structure of similar size and strength, albeit of the opposite magnitude, appears (also below the flame surface) to the right of the centerline and likewise propagates up through the field of view. Based on the POD analysis described earlier and the timescale over which this pair of vortices appear $(\sim 2 \mathrm{~ms})$, it is clear these structures represent planar slices of the three-dimensional PVC structure.

The effect of the PVC on the reaction zone in this image sequence is clear. The reaction zone conforms to the passage of the large-scale vortical structures as they appear and move downstream. As noted previously, the flame sheet remains in the low-velocity periphery of the vortices, but is clearly influenced by the passage of the structures. In frames 1-7, the reaction zone moves upstream and to the left, elongating and wrapping around the vortex. By frame 6 , the center of the vortex has moved several millimetres from the reaction zone and has decreased in diameter, suggesting that heat-release and dilatation around the flame has diminished the strength of the vortex. In frames 7 through 11, a similar movement of the reaction zone relative to the vortex structure to the right side of the vertical axis is observable. In this instance, however, the motion of the reaction zone is complicated by the appearance of several isolated pockets of $\mathrm{OH}$ upstream of the contiguous reaction zone, which grow and interact with the local flow-field. 
The image sequence shows the shape and instantaneous spatial position of the reaction zone is significantly affected by the local dynamics of both the PVC and the thermo-acoustic oscillation. Whereas the thermo-acoustic oscillation is a dominant parameter affecting axial velocity fluctuations and thus the absolute axial location of the reaction zone, the tendency of the flame to conform to the passage of large-scale vortices indicates the shape or wrinkledness of the flame is significantly affected by the PVC. Although Stöhr and Meier [2007] determined the principal motion of the PVC does not correlate to the thermo-acoustic oscillation, the simultaneous effect of both on the shape and location of the reaction zone, together with the stabilizing effect of local heat-release by a flame suggests the two are locally coupled near the nozzle exit. The secondary peaks observed in the velocity spectra at the jetand shear-layer monitor are consistent with a local coupling between the PVC and thermoacoustic oscillation.

\section{$\underline{\text { Local Extinction }}$}

In frame 11 of the image sequence, a break appears in the reaction zone at approximately $7 \mathrm{~mm}$ to the left of centreline, and $14 \mathrm{~mm}$ downstream of the exit plane. Although the exact size and centerpoint of the break will inevitably be sensitive to the magnitude of the threshold used to determine reaction zone location from the $\mathrm{OH}$ gradient field, it is nonetheless clear that this break is physical, spatially significant and representative of local extinction of the reaction zone. Evidence of local flame extinction has been noted by previous researchers [Meier et al., (2006)], but the dynamics of the process have yet to be fully explored.

The reason for this sudden breach in the reaction zone is not immediately clear from the velocity or vorticity data. A small region of high axial-velocity fluid is visible near the flame hole in frame 11, but it remains largely overlapped with the flame-sheet rather than the breach, indicating that fluid exceeding the local flame propagation speed is not a likely explanation. Similarly, although through-plane velocity is an important parameter governing Boxx et al., Combust. Flame (2010). Accepted for Publication Dec. 16, 2009. 
the dynamics of this flame, the breech first appears in a region of relatively low Vz-magnitude fluid, indicating it is not likely to have been swept in from beyond the measuring plane. There is no evidence of a large-scale vortical structure in the immediate vicinity of the breach, indicating elongation and distortion of the reaction zone by the PVC does not explain the extinction either. Indeed, as the present measurements do not include temperature or mixture fraction, it is impossible to conclusively determine the cause of this event. The data do, however, yield a promising explanation.

Shown in Figure $8 \mathrm{f}$ is the reaction zone contour overlaid against contours of minimum 2D principal normal strain. Minimum 2D principal normal strain is perhaps the most relevant flow-field metric available in this data set as previous experimental [Rehm and Clemens, (1998); Kothnur and Clemens, (2005)] and numerical [Ashurst et al., (1987)] studies indicate the largest scalar gradients tend to align with the axis of principal compressive strain and the strongest dissipation layers form orthogonal to this axis. Local extinction in a turbulent diffusion flame is frequently observed when a strong scalar dissipation layer crosses the stoichiometric contour [Sutton and Driscoll, (2007)]. The thinnest reaction zones are also to be found orthogonal to the axis of principal compressive strain [Rehm and Clemens (1998)]. Beginning at frame 6 , a region of large magnitude $\left(>2000 \mathrm{~s}^{-1}\right)$ principal normal strain forms upstream of the reaction zone, near the burner exit. This high strain region remains close to the reaction zone throughout the subsequent frames until extinction occurs in frame 11 . During this period, the reaction zone is observed to re-orient itself, aligning parallel to the major axis of this high strain region. In frame 10, the high strain region crosses the reaction zone at the location where the reaction zone breech will occur in the next frame. It is well established [Rehm and Clemens (1998)] that turbulent flames frequently experience transient strains significantly exceeding their steady state extinction values while remaining intact. Böhm et al. [2009] used spatio-temporal conditioning of simultaneous time-resolved PIV and 
OH PLIF in a turbulent opposed jet flame to show that extinction occurs only after the strain near the reaction zone exceeds a critical value for a sufficient length of time. In the present sequence, the region of high principal compressive strain is seen to persist behind the reaction zone over five frames, or approximately one millisecond. This, taken together with the lack of high axial-velocity and / or vorticity fluid in the immediate vicinity of the breach, suggests this region of high $2 \mathrm{D}$ principal normal strain is the primary cause of the local flame extinction. Referring back to frame 10 of Fig. 8c, this region of high principal compressive strain results from the appearance of high axial-velocity fluid near the exit, which then impacts the flame sheet. As one sees in Fig. 8e, the flame sheet simultaneously elongates and conforms to the passage of a large-scale vortical structure to the right of the centreline. Together, the combination of high axial-velocity unburned gas with a downward propagating reaction zone results in stagnation point flow. Thus, it appears both the strong axial-velocity oscillation associated with thermo-acoustic pulsation and the movement of the reaction zone as it conforms to the passage of the PVC in this case combine to generate a high strain stagnation point flow which persists over a sufficiently long period to extinguish the flame locally.

\section{Isolated $\mathrm{OH}$ Pockets}

A frequently observed phenomenon in the measurement sequences was the sudden appearance of isolated pockets of $\mathrm{OH}$ in regions of unburned gas significantly upstream of the contiguous flame-front. Such pockets, for example, appear in frames 7 and 15 of the image sequence described above. These pockets of $\mathrm{OH}$ either grow in size over the next several frames before coalescing with the contiguous reaction zone, or rapidly collapse and disappear. For the case where these $\mathrm{OH}$ pockets grow, they tend to do so very rapidly, forming highly corrugated reaction zones. As one can see for the case of the expanding $\mathrm{OH}$ pocket appearing in frame 7 , there is a rapid increase in reaction zone length about the periphery of such 
pockets. As heat release occurs primarily in the vicinity of the reaction zones, the sudden appearance and rapid expansion of these $\mathrm{OH}$ pockets may represent an additional mechanism of flame stabilization in this burner.

The appearance of isolated pockets of $\mathrm{OH}$ in the measurement region can result from one of two mechanisms, auto-ignition of the fuel-air mixture in a region of unburned gas or propagation and/or convection of a flame sheet into the field of view from out of plane. The tendency of turbulent flames to form long 'fingers', which may pinch off and burn out separately from the contiguous flame sheet has been observed in turbulent premixed flames (Filatyev et al., [2005]). Viewed in a plane perpendicular to such a "flame finger", the flame would appear as an isolated OH-pocket. As the PLIF technique identifies only the presence of $\mathrm{OH}$ and not its source, it is impossible to definitively conclude which mechanism is responsible for the appearance of such a pocket in any given instance. The extensive dynamic fluctuation of the (in-plane) reaction zone together with the high through-plane velocities present in the swirling flow indicate that the latter mechanism is a convincing explanation for the sudden appearance of these pockets some of the time. Indeed, the $\mathrm{OH}$ pocket seen in frame 7 of the measurement sequence appears in a region of relatively high into-plane velocity, suggesting it may have been transported into the field of view. Evidence from the present measurements and previous studies indicate, however, that auto-ignition is also a viable explanation.

Figure 9 shows a scatter-plot of the mixture fraction vs. temperature based on singleshot Raman data of Meier et al. [2006] at approximately the same location under identical flame conditions. Each symbol in the figure represents the result of a single shot measurement, and the line displays the relation for adiabatic equilibrium conditions. There is a significant variation in mixture fraction ranging from 0 (pure air) to about 0.15 (equivalence ratio $\Phi \approx 3$ ). The scatter in temperature reflects the different thermo-chemical states: nonBoxx et al., Combust. Flame (2010). Accepted for Publication Dec. 16, 2009. 
reacted mixtures at room temperature, completely reacted mixtures with $\mathrm{T}$ close to $\mathrm{T}_{\mathrm{ad}}$ (probably stemming from the IRZ) and partially reacted samples with intermediate temperatures. The partially reacted mixtures mostly reflect mixtures of burned gas from the recirculation zones and fresh gas from the nozzle which have not yet reacted. Particularly the mixtures exhibiting elevated temperatures can be subject to auto-ignition. Meier et al. [2006] calculated the ignition delay for mixtures of cold $\mathrm{CH}_{4}$-air and adiabatic exhaust gas and found it be on the order of $\mathrm{t}<0.5 \mathrm{~ms}$ and largely insensitive to mixture fraction for temperatures near detection limit of the OH PLIF system, approximately $1500 \mathrm{~K}$. Inspection of the axialvelocity and vorticity fields for this sequence shows the $\mathrm{OH}$ pockets in frame 7 first appear near the center of the large vortex structure propagating up and to right of the centreline in that frame. The vectors and axial velocity contours in Fig. 8c indicate low absolute velocity magnitude in the region of the $\mathrm{OH}$ pocket. Tracing the progress of the vortex through earlier frames indicates that fluid near the core may experience residence times on the order of a millisecond or more, i.e. greater than the auto-ignition delay, provided the local fluid temperature is sufficiently high. It was noted previously that the reaction zone conforms to the passage of this large scale vortex, indicating that fluid at the core may experience significant pre-heating resulting from mixing induced by the vortex. Taken together, these data indicate auto-ignition is a viable explanation for the sudden appearance of this $\mathrm{OH}$ pocket.

The appearance of isolated $\mathrm{OH}$ regions near the center of large-scale vortical structures in the unburned gas occurred frequently throughout each measurement run. The $\mathrm{OH}$ pocket identified in frame 15, for example, also appears near the core of a large scale vortex. Figure 10 shows a similar measurement sequence acquired during the same imaging run. The data in this sequence shows the reaction zone overlaid against a vorticity field determined from velocity data without application of the bandpass filtered POD reconstruction or interpolation beyond single vector gaps in the vector post-processing algorithms. As expected, 
this sequence is noisier than the previous one, but the physical interpretation remains the same. In frame 2 an isolated pocket of $\mathrm{OH}$ appears in the measurement plane close to a largescale vortical structure and in a region of unburned gas away from the contiguous reaction zone. The increased noise makes it difficult to determine how close this $\mathrm{OH}$ pocket is to the center of the vortex when it appears, but it does nonetheless appear to be associated with the structure. This region of $\mathrm{OH}$ grows rapidly and soon engulfs the vortical structure before coalescing with the contiguous reaction zone. As in the previous example, the appearance of this $\mathrm{OH}$ pocket results in a rapid increase in reaction zone area present in the measurement region.

\section{Conclusions}

Stereoscopic PIV and OH PLIF were applied simultaneously at a repetition rate of $5 \mathrm{kHz}$ to study the dynamics and turbulence-chemistry interaction of a $10 \mathrm{~kW}$, swirl-stabilized, methane-air flame exhibiting self-excited thermo-acoustic oscillation. Spatial and temporal frequency content were extracted via time-series analysis. Interpretation of individual measurement sequences yielded physical insight into various relevant combustion phenomena and the underlying mechanisms driving flame dynamics.

The reaction zone was seen to reside primarily in the regions of low axial velocity fluid along the interface between incoming unburned gas and the high-temperature inner recirculation zone (IRZ). The axial position of the flame front was seen to track largemagnitude axial-velocity fluctuations, while remaining in locally low speed regions of the flow. The reaction zone also conforms to the passage of large scale vortex structures associated with a precessing vortex core present within the flow-field. This resulted in significant wrinkling and corrugation of the reaction zone. 
Local extinction of the reaction zone occurred frequently along the interface between the incoming gas and IRZ. One mechanism for such extinctions appeared to be high 2D principal compressive strain in the immediate vicinity of the reaction zone that persists over timescales $>1 \mathrm{~ms}$. Under the flow conditions of this study, local extinctions are short-lived and the flame surface is observed to mend quickly after such events. Regions of unburned gas are frequently observed beyond the contiguous reaction zone, resulting from the engulfment of fluid by the flame during large-scale fluctuations of the IRZ. These regions burn out as they propagate downstream.

Isolated regions of $\mathrm{OH}$ appeared frequently in regions of unburned gas away from the contiguous reaction zone. The evidence indicates that auto-ignition is a reasonable explanation for at least some of these events. The rapid expansion of reaction zone length associated with the growth of these flame kernels indicates that they could have a significant impact on the instantaneous local heat-release rate of the flame. Further, they present an additional contribution to the stabilization of the flame and, by extension, the driving mechanism of the thermo-acoustic oscillation.

The results presented also demonstrate that the high-speed planar diagnostics system is capable of providing good quality OH PLIF and PIV measurements at sustained repetition rates of $5 \mathrm{kHz}$, enabling the recording of the temporal development of the interaction between the flow-field and the flame-fronts. In this way, the dynamic processes within a GT-relevant swirl flame could be visualized for the first time with sufficient temporal resolution. As demonstrated, the results clearly help identify the sequence of events leading to phenomena like flame-front deformation or local flame extinction. With respect to periodic instabilities, the time series of roughly 4000 correlated images easily allow the determination of the dominant frequencies. In addition, the data sets allow for conditional averaging or averaging with respect to the phase of the thermo-acoustic or hydrodynamic instability (PVC). In the ongoing data evaluation, it is intended to analyze, for example, the influence of the acoustic Boxx et al., Combust. Flame (2010). Accepted for Publication Dec. 16, 2009. 
phase on local flame extinction events or on the appearance of isolated $\mathrm{OH}$ pockets near the nozzle.

Despite the tremendous gain in temporal resolution for simultaneous OH PLIF and stereo PIV measurements compared to conventional low-repetition systems, some ambiguities remain in the interpretation of the results stemming from the out-of-plane motion, particularly in swirl flames. As described, the frequently measured $\mathrm{OH}$ pockets, which are isolated within the imaged area, might be connected to a larger contiguous flame surface located behind or in front of the interrogation plane. For an unambiguous identification of auto-ignition events in this flame, it would be necessary to apply one or two additional OH PLIF sheets, either parallel or inclined to the original one, and thus this is an area for future work. 


\section{References}

B.O. Ayoola, R. Balachandran, J.H. Frank, E. Mastorakos, C.F. Kaminski. Comb. Flame, 144 (2006) 1-16.

R.J. Adrian, K.T. Christensen, Z.-C. Liu, Exp. Fluids 29 (2000) 275-290.

P.M. Anacleto, E.C. Fernandes, M.V. Heitor, S.I. Shtork, Combust. Sci. And Technol. 175 (2003) 1369-1388.

W.T. Ashurst, A.R. Kerstein, R.M. Kerr, C. H. Gibson, Phys. Fluids 30, (1987), 2343 - 2353.

H. Ax, U. Stopper, W. Meier, M. Aigner, F. Güthe, Proceedings of ASME Turbo Expo 2009, GT2009-59171.

R. Balachandran, B.O. Ayoola, C.F. Kaminski, A.P. Dowling, E. Mastorakos. Combust. Flame, 143 (2005) 37-55.

B.D. Bellows, M.K. Bobba, A. Forte, J.M. Seitzman, T. Lieuwen. Proc. Comb. Inst. 31 (2007) 3181-3188.

G. Berkooz, P. Holmes, J.L. Lumley, Ann. Rev. Fluid Mech., (1993) 539-575.

B. Böhm, I. Boxx, C. Kittler, W. Meier, A. Dreizler. Proc. Comb. Inst. 32 (2009) 1647-1654.

I. Boxx, M. Stöhr, R. Blumenthal, C. Carter, W. Meier, AIAA 2009-644, 47th AIAA Aerospace Sciences Meeting, 5-8 January (2009a).

I. Boxx, C. Heeger, R. Gordon, B. Böhm, A. Dreizler, W. Meier, Combust. Flame, 156 (2009b) 269-271.

I. Boxx, M. Stöhr, C. Carter, W. Meier, Appl. Phys B, 95(1) 2009c. 23-29.

S. Candel. Proc. Comb. Inst. 29 (2002) 1-28.

S. Correa. Proc. Comb. Inst. (1998) 1793-1807.

N. Docquier, S. Candel, Prog. Energy Combust. Sci. 28 (2002) 107-150.

X. R. Duan, W. Meier, P. Weigand, B. Lehmann, Appl. Phys. B 80 (2005) 389-396.

I. Emiris, J.H. Whitelaw, Combust. Sci. Technol. 175 (2003) 157-184.

C. Fajardo, V. Sick, Exp. Fluids 46, (2009) 43-53.

E.C. Fernandes, M.V. Heitor, in F. Culick, M.V. Heitor, J.H. Whitelaw (Eds.), Unsteady Combustion, Kluwer Academic press, Dordrecht, 1996.

S.A. Filatyev, J.F. Driscoll, C.D. Carter, J.M. Donbar, Combus. Flame 141, (2005) 1-21.

D. Froud, T. O’Doherty, N. Syred, Combust. Flame, 100 (1995) 407-412. 
R. Giezendanner-Thoben, O. Keck, P. Weigand, W. Meier, U. Meier, W. Stricker, M. Aigner, Combust. Sci. Tech. 175 (2003) 721-741.

R. Giezendanner-Thoben, P. Weigand, X. R. Duan, W. Meier, U. Meier, M. Aigner, B. Lehmann, J. Eng. Gas Turb. Power. 127 (2005a) 492-496.

R. Giezendanner-Thoben, U. Meier, W. Meier, M. Aigner, "Phase-Locked Temperature Measurements by Two-Line OH PLIF Thermometry of a Self-Excited Combustion Instability in a Gas Turbine Model Combustor", Flow Turb. Combust. 75 (2005b) 317-333.

R. Giezendanner-Thoben, U. Meier, W. Meier, J. Heinze, M. Aigner, Appl. Opt. 44 (2005c) $6565-6577$

J.R. Gord, M.S. Brown, T.R. Meyer, 22nd AIAA Aerodynamic Measurement Technology and Ground Testing Conference, 24-26 June 2002, St. Louis, Missouri, paper AIAA 2002-3039

P. Griebel, P. Siewert, P. Jansohn, Proc. Combust. Inst. 31, (2007) 3083-3090.

A. K. Gupta, D.G. Lilley, N. Syred, Swirl Flows, Abacus Press (1984)

W.A. Guttenfelder, M.W. Renfro, N.M. Laurendeau, J. Ji, G.B. Galen, J.P. Gore, Combust. Flame 147 (2006) 11-21.

J. Hult, U. Meier, W. Meier, A. Harvey, C.F. Kaminski, Proc. Combust. Inst. 30 (2005) 701709.

B. Janus, A, Dreizler, J. Janicka, Proc. Comb. Inst. 31 (2007) 3091-3098.

J. Ji, J.P. Gore, Proc. Combust. Inst. 29 (2002) 861-867.

J.J. Keller, AIAA J. 33 (1995) 2280-2287.

J. Kojima, Q.-V. Nguyen, Meas. Sci. Technol. 15 (2004) 565-580.

M. Konle, T. Sattelmayer, Exp. Fluids. (In Print) DOI 10.1007/s00348-009-0679-5.

M. Konle, F. Kiesewetter, T. Sattelmayer, Exp. Fluids 44 (2008) 529-538.

P.S. Kothnur, N.T. Clemens, Phys. Fluids 17 (2005) 1-14.

S.-Y. Lee, S. Seo, J.C. Broda, S. Pal, R.J. Santoro, Proc. Combust. Inst. 28 (2000) 775-782.

J.G. Lee, D.A. Santavicca, J. Propulsion and Power 19 (2003) 735-750.

A.H. Lefebvre, Gas Turbine Combustion, Taylor \& Francis, Philadelphia, 1999

T. Lieuwen, V. Yang. Combustion Instabilities in Gas Turbine Engines: Operational Experience, Fundamental Mechanisms, And Modeling, AIAA (2005). 
C. Löfström, J. Engström, M. Richter, C.F. Kaminski, P. Johansson, K. Nyholm, J. Nygren, M. Aldén, Proc. ASME Turbo Expo 2000, 2000-GT-0124, May 8-11, 2000, Munich, Germany

O. Lucca-Negro, T. O'Doherty, Vortex breakdown: a review, Prog. Energy Combust. Sci. 27 (2001) 431-481.

U.E. Meier, D. Wolff-Gaßmann, W. Stricker, Aerosp. Sci. Technol. 4, (2000) 403-414.

W. Meier, X.R. Duan, P. Weigand, Proc. Combust. Inst. 30, (2002) 835-842.

W. Meier, X. R. Duan, P. Weigand, Combust. Flame 144 (2006) 225-236.

H.C. Mongia, T.J. Held, G.C. Hsiao, R.P. Pandalai, J. Propulsion and Power 19 (2003) 822829.

T.R. Meyer, G.J. Fiechtner, S.P. Gogineni, J.C. Rolon, C.D. Carter, J.R. Gord, Exp. Fluids 36 (2004) 259-267.

C.O. Paschereit, E. Gutmark, W. Weisenstein, Combust. Sci. Technol. 138 (1998) 213-232.

P. Petersson, J. Olofsson, C. Brackmann, H. Seyfried, J. Zetterberg, M. Richter, M. Alden, M.A. Linne, R.K. Cheng, A. Nauert, D. Geyer, A. Dreizler, Appl. Opt. 46, (2007) 3928-3936.

J.E. Rehm, N.T. Clemens. Proc. Combust. Inst. 27 (1998) 1113-1120.

G.A. Richards, D.L. Straub, E.H. Robey, J. Propulsion Power 19 (2003) 795-810.

S. Roux, G. Lartigue, T. Poinsot, U. Meier, C. Berat, Comb. Flame, 121, (2005) 40-54.

R. Sadanandan, M. Stöhr, W. Meier, Appl. Phys. B 90 (2008) 609-618.

K.-U. Schildmacher, R. Koch, H.-J. Bauer, Flow, Turb. and Combust. 76 (2006) 177-197.

A.M. Steinberg, J.F. Driscoll, S.L. Ceccio, Exp Fluids 44 (2008) 985-999.

M. Stöhr, W. Meier, 12th international symposium on flow visualization (2006)

M. Stöhr, W. Meier, Proc. Europ. Combust. (2007)

M. Stöhr, R. Sadanandan, W. Meier, Proc. Comb. Inst. 32 (2009) 2925-2932.

L. Sirovich. Q. Applied Mathematics. XLV:3 (1987) 561-571.

U. Stopper, M. Aigner, W. Meier, R. Sadanandan, M. Stöhr, I.S. Kim, Proc. of 2008 ASME Turbo Expo, GT2008-50520

J.A. Sutton, J.F. Driscoll. Proc. Combust. Inst. 31 (2007), 1487-1495.

P.A. Strakey, S.D. Woodruff, T.C. Williams, R.W. Schefer, AIAA J. 46 (2008) 1604-1613. 
N. Syred, N.A. Chigier, J.M. Beér, Proc. Combust. Inst. 13 (1971), 617-624.

N. Syred, Prog. Energy Combust. Sci. 32 (2006) 93-161.

M. Tanahashi, S. Murakami, G.-M. Choi, Y. Fukuchi, T. Miyauchi, Proc. Combust. Inst. 30, (2005) 1665-1672.

S.K. Thumuluru, T. Lieuwen. Proc. Combust. Inst. 32, (2009) 2893-2900.

A. Upatnieks, J.F. Driscoll, C.C. Rasmussen, S.L. Ceccio, Comb. Flame 138 (2004) 259-272.

A. Valera-Medina, N. Syred, A. Griffiths, Comb. Flame 156 (2009) 1723-1734.

C.M. Vagelopoulos, J.H. Frank, Combust. Flame 146 (2006) 572-588.

K.K. Venkataraman, L.H. Preston, D.W. Simons, B.J. Lee, J.G. Lee, D.A. Santavicca. J. Prop. Power 15:6 (1999) 909-918.

S. Wang, S-Y. Hsieh, V. Yang. Phys. Fluids, 17 (2005), 045106.

R. Weber, J. Dugué, Prog. Energy Combust. Sci. 18 (1992) 349-367.

B. Wegner, A. Maltsev, C. Schneider, A. Sadiki, A. Dreizler, J. Janicka. Int.J.Heat and Fluid Flow 25, (2004) 528-536.

P. Weigand, W. Meier, X. R. Duan, R. Giezendanner-Thoben, U. Meier, Flow Turbul. Combust. 75 (2005) 275-292.

P. Weigand, W. Meier, X. R. Duan, W. Stricker, M. Aigner, Investigations of swirl flames in a gas turbine model combustor I. Turbulence-chemistry interactions, Combust. Flame 144 (2006) 205-224.

A. Widenhorn, B. Noll, M. Aigner, AIAA 2009-647, 47th AIAA Aerospace Sciences Meeting, 5-8 January 2009.

C. Willert, C. Hassa, G. Stockhausen, M. Jarius, M. Voges, J. Klinner, Meas. Sci. Technol. 17 (2006) 1670-1679.

\section{Acknowledgments}

Campbell Carter acknowledges support of the Air Force Office of Scientific Research (AFOSR) Windows on Europe Program. 


\section{Figure Captions}

Figure 1. a) Experiment configuration. b) Dual-swirl GTMC burner

Figure 2. a) Mean centerline flow-field for the burner chamber. b) Mean flow-field within imaged region, including monitor points used for frequency analysis.

Figure 3. a) Sample OH PLIF image. b) OH gradient magnitude

Figure 4. Power spectra of axial velocity in a) Inner shear layer (ISL) b) Inner recirculation zone (IRZ) c) jet. Power spectra of d) integrated chemiluminescence and e) acoustic signal

Figure 5. Power spectra of POD temporal eigenmodes a) first, b) second and c) third

Figure 6. Spatial eigenmodes 1, 2 and 3 of POD. Modes 1 and 2 correspond to the PVC, while 3 corresponds to the thermo-acoustic pulsation.

Figure 7. Measured acoustic signal corresponding to sample sequence shown in Fig. 8.

Figure 8. a) OH PLIF images - time separation $=0.2 \mathrm{~ms}$

Figure 8. b) OH Gradient images. Large magnitude gradients indicate reaction zones.

Figure 8. c) Reaction zones overlaid against contours of axial velocity. For clarity, only every second computed vector is plotted in the vector overlay.

Figure 8. d) Reaction zones overlaid against contours of Vz- (through plane) velocity. For clarity, only every second computed vector is plotted in the vector overlay.

Figure 8 . e) Reaction zones overlaid against contours of vorticity

Figure 8. f) Reaction zones overlaid against contours of minimum principle normal strain

Figure 9. Scatter plot of data acquired by Meier et al. [2006] showing evidence of auto ignition around location where isolated $\mathrm{OH}$ pockets appear in the image sequences.

Figure 10. Development of isolated $\mathrm{OH}$ pocket (first evident near $\mathrm{x}=-5 \mathrm{~mm}$ in frame 2), overlaid on unfiltered vorticity data. 


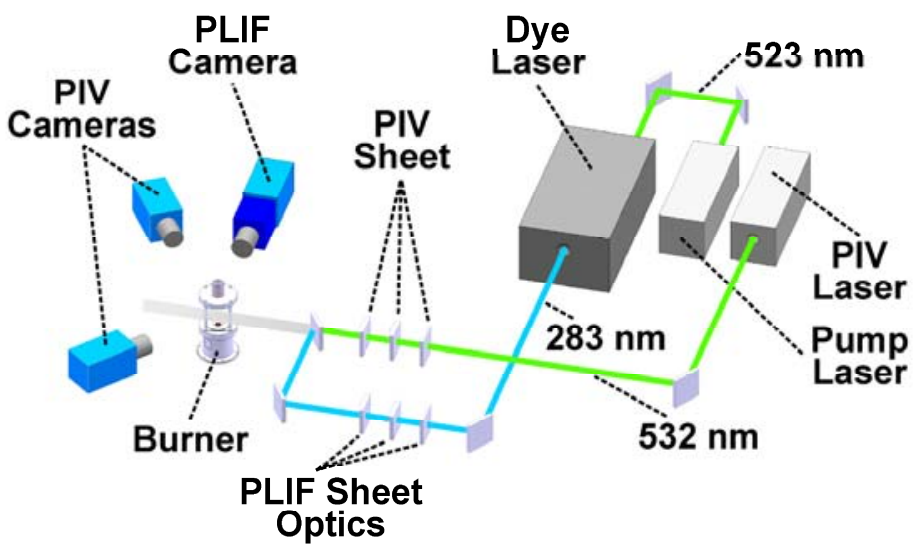

(a)
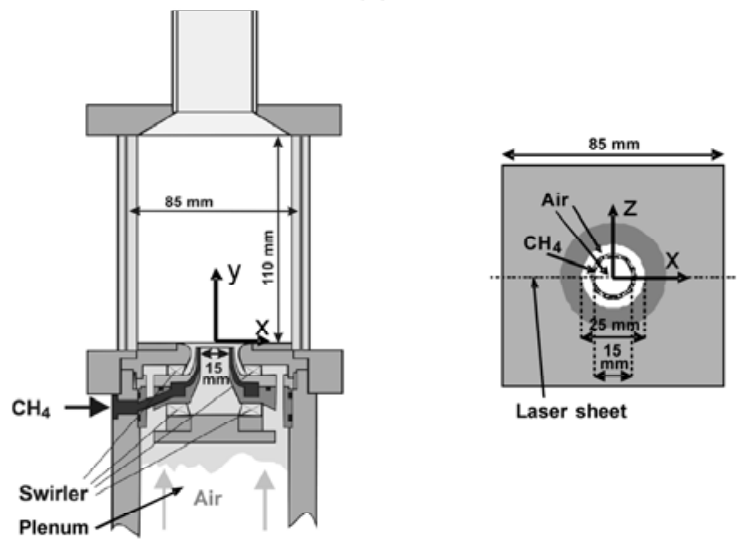

Side view

(b)

Top view

b)

Figure 1. a) Experiment configuration. b) Dual-swirl GTMC burner 


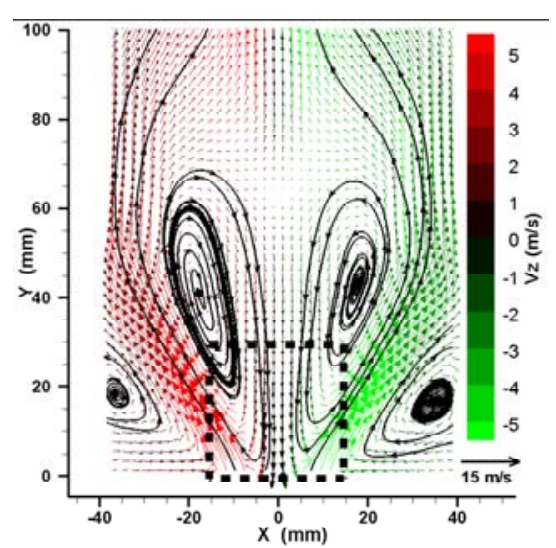

(a)

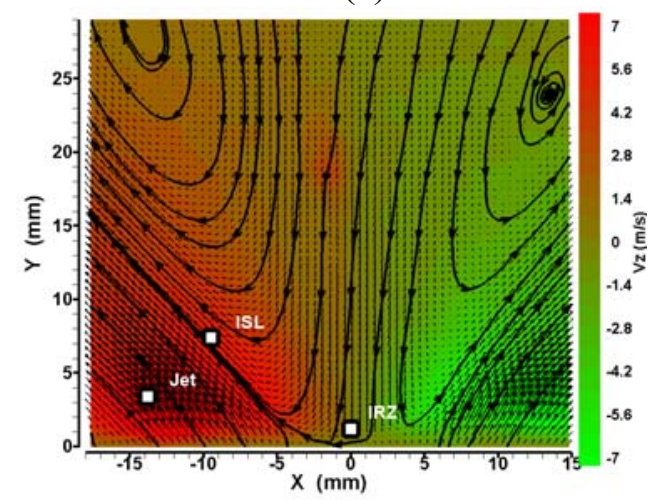

(b)

Figure 2. a) Mean centerline flow-field for the burner chamber. b) Mean flow-field within imaged region, including monitor points used for frequency analysis.

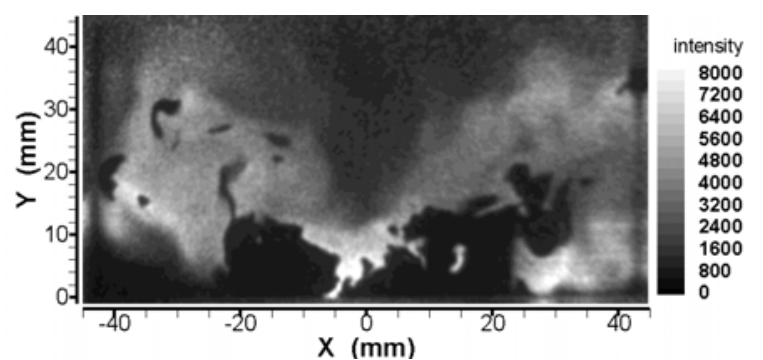

(a)

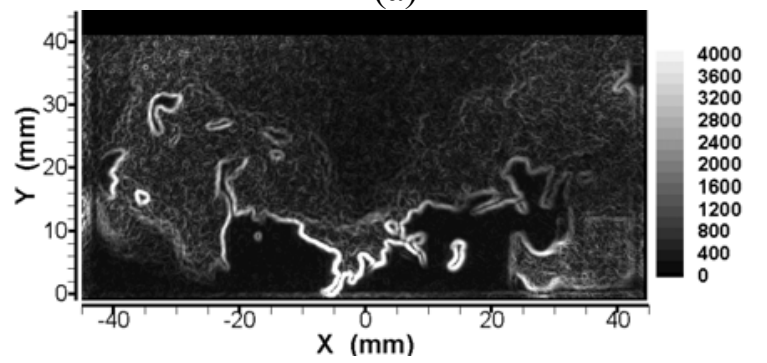

(b)

Figure 3. a) Sample OH PLIF image. b) OH gradient magnitude 


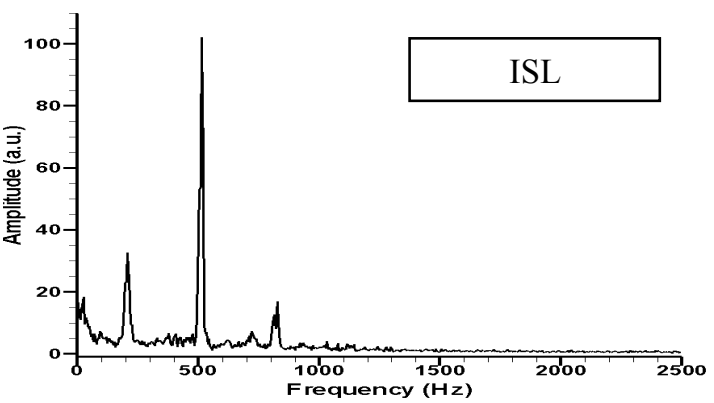

(a)

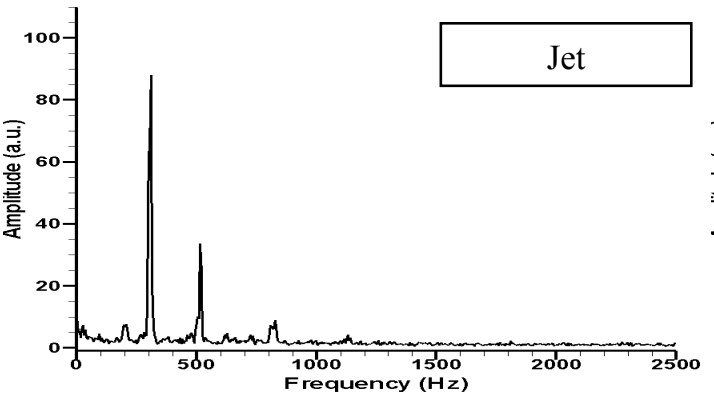

(c)

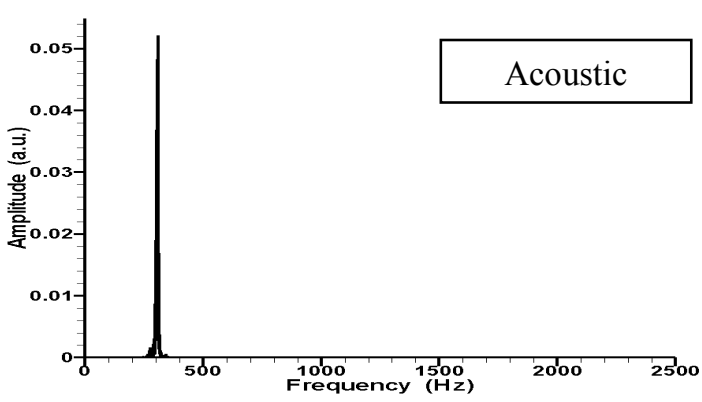

(e)

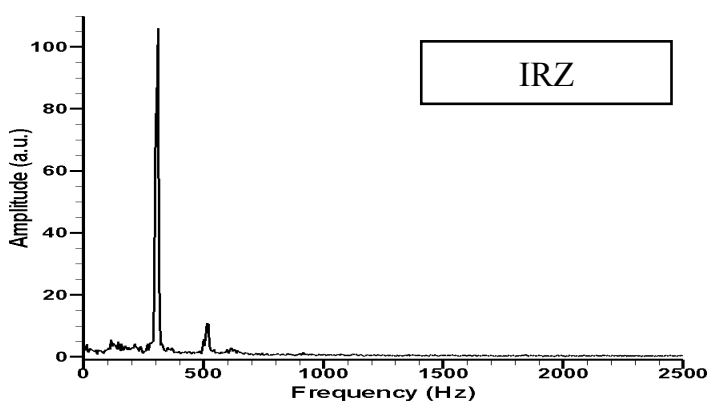

(b)

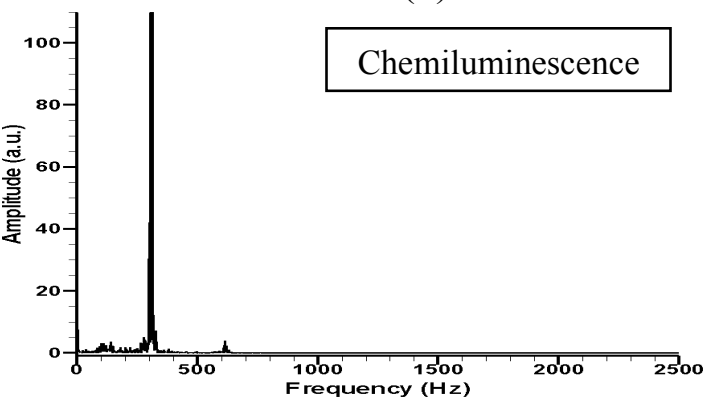

(d)

Figure 4. Power spectra of axial velocity in a) Inner shear layer (ISL) b) Inner recirculation zone (IRZ) c) jet. Power spectra of d) integrated chemiluminescence and e) acoustic signal 


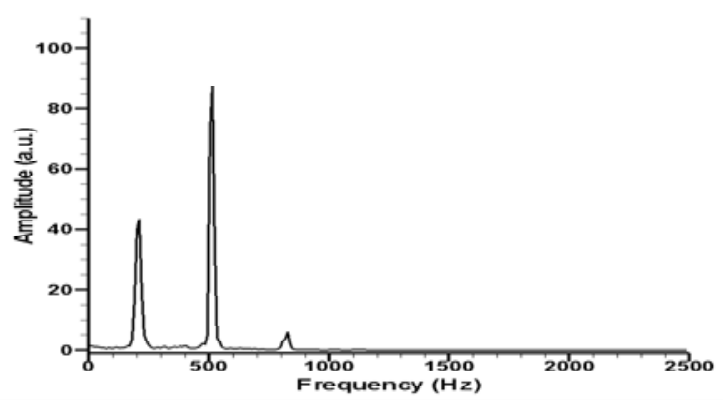

(a)

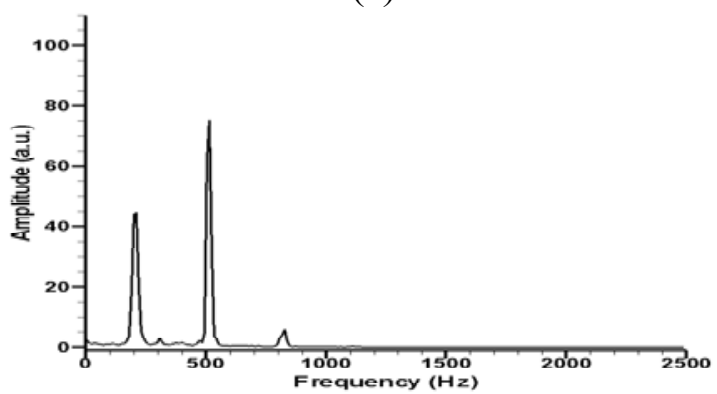

(b)

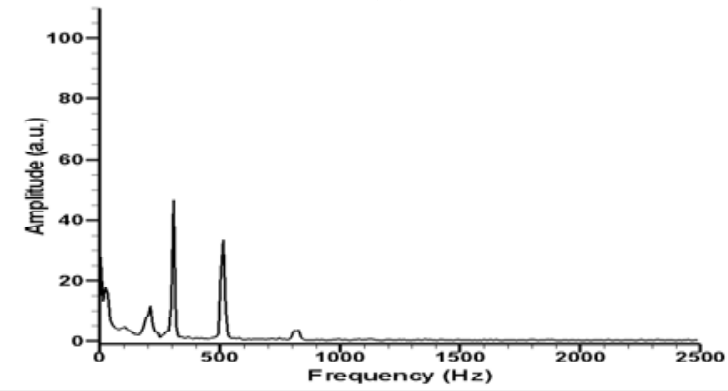

(c)

Figure 5. Power spectra of POD temporal eigenmodes a) first, b) second and c) third 


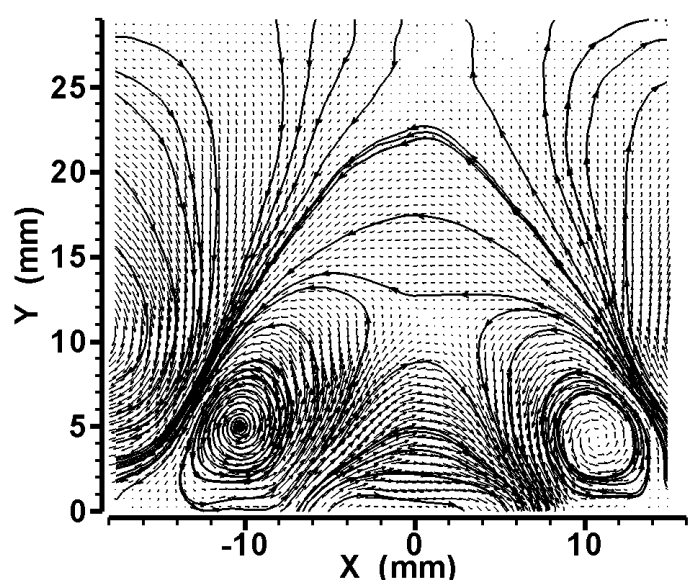

(a)

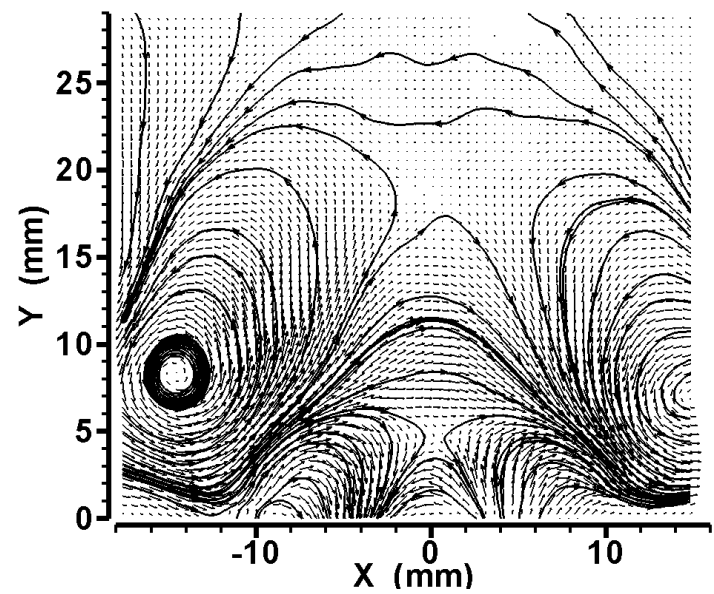

(b)

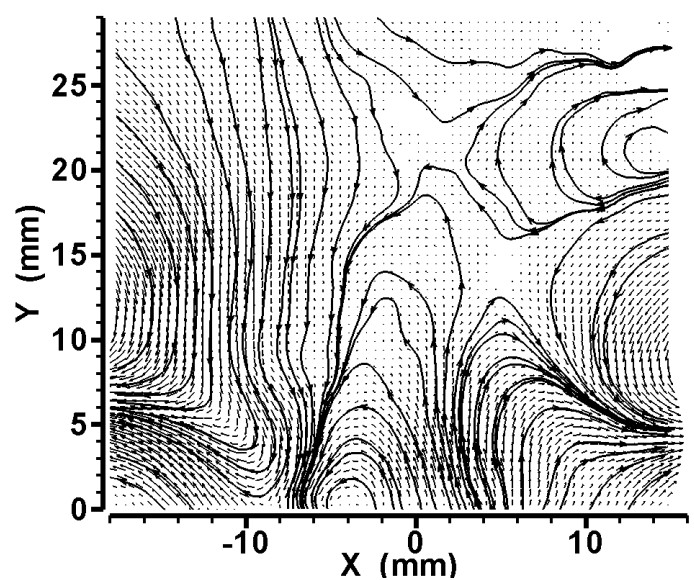

(c)

Figure 6 . Spatial eigenmodes 1, 2 and 3 of POD. Modes 1 and 2 correspond to the PVC, while 3 corresponds to the thermo-acoustic pulsation. 


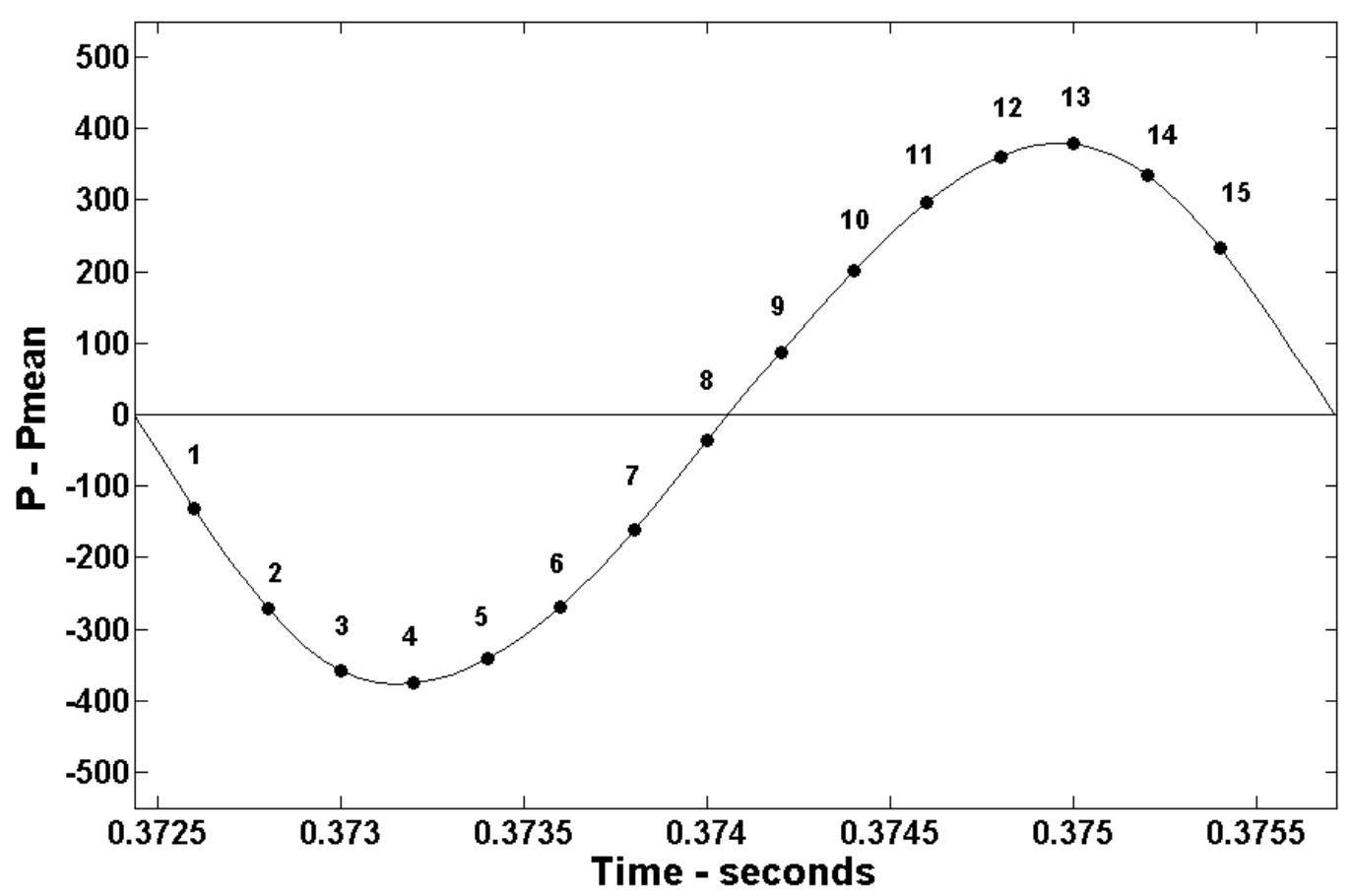

Figure 7. Spatial eigenmodes 1, 2 and 3 of POD. Modes 1 and 2 correspond to the PVC, while 3 corresponds to the thermo-acoustic pulsation. 

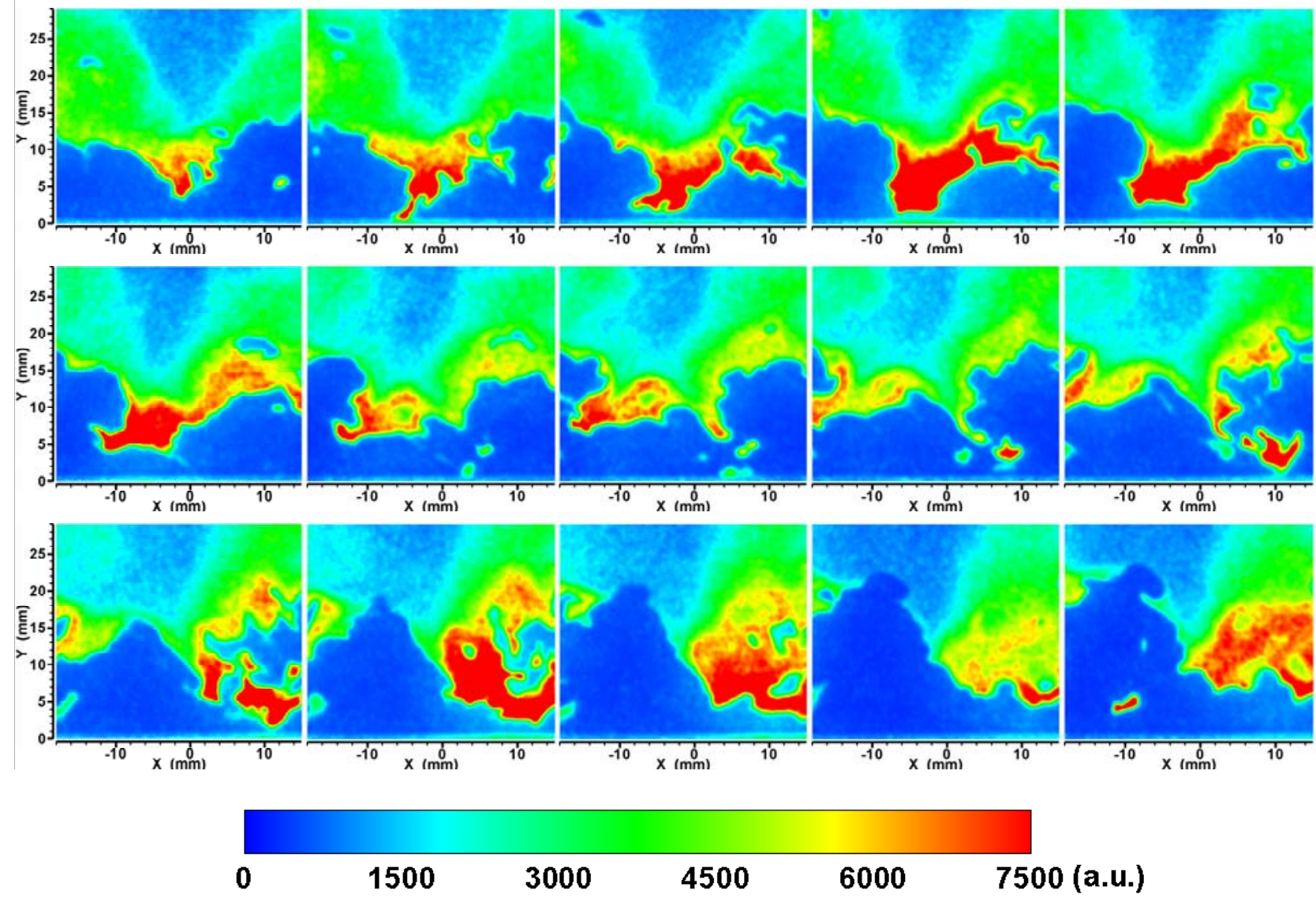

Figure 8. a) OH PLIF images - time separation $=0.2 \mathrm{~ms}$ 

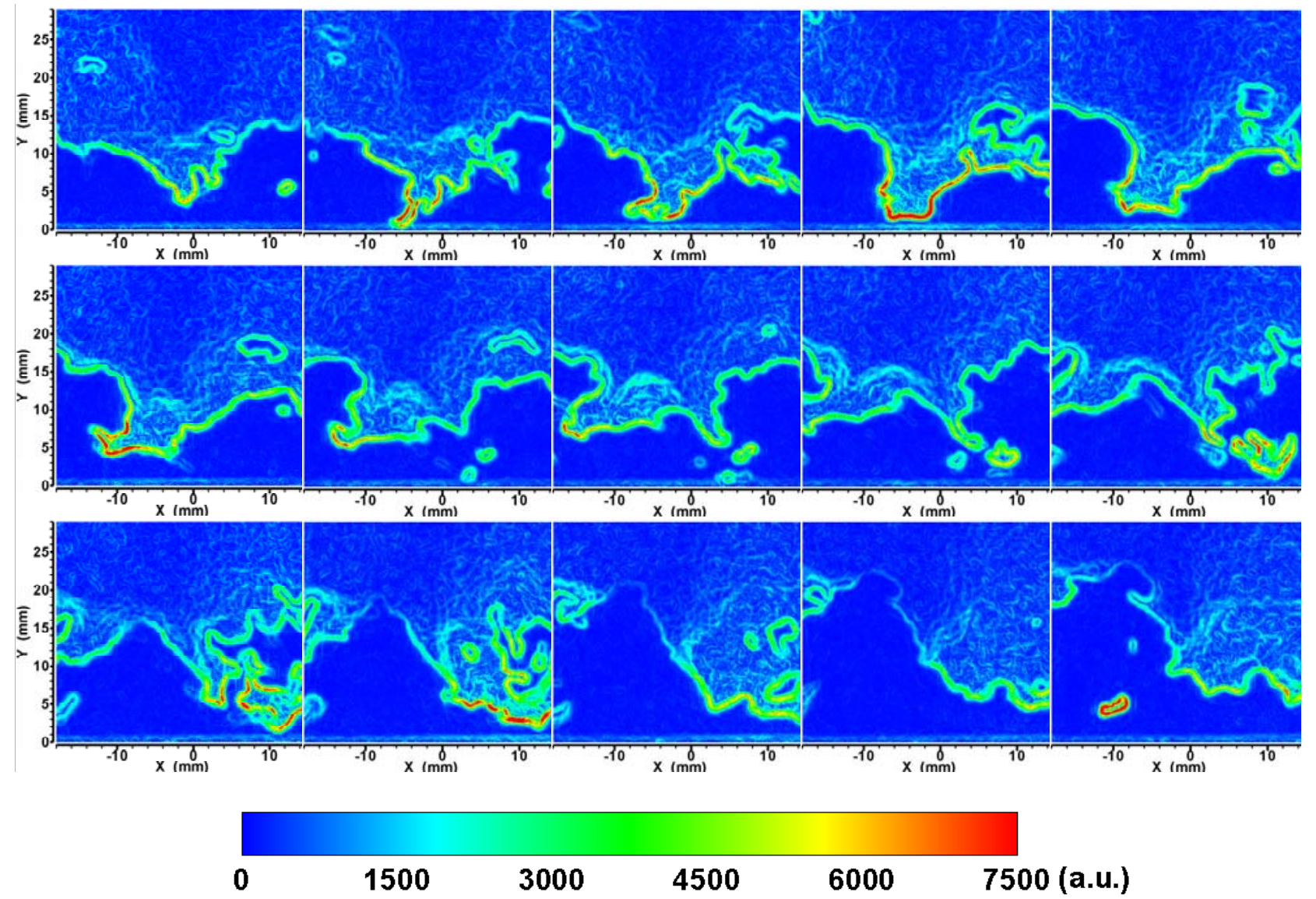

Figure 8. b) $\mathrm{OH}$ Gradient images. Large magnitude gradients indicate reaction zones. 

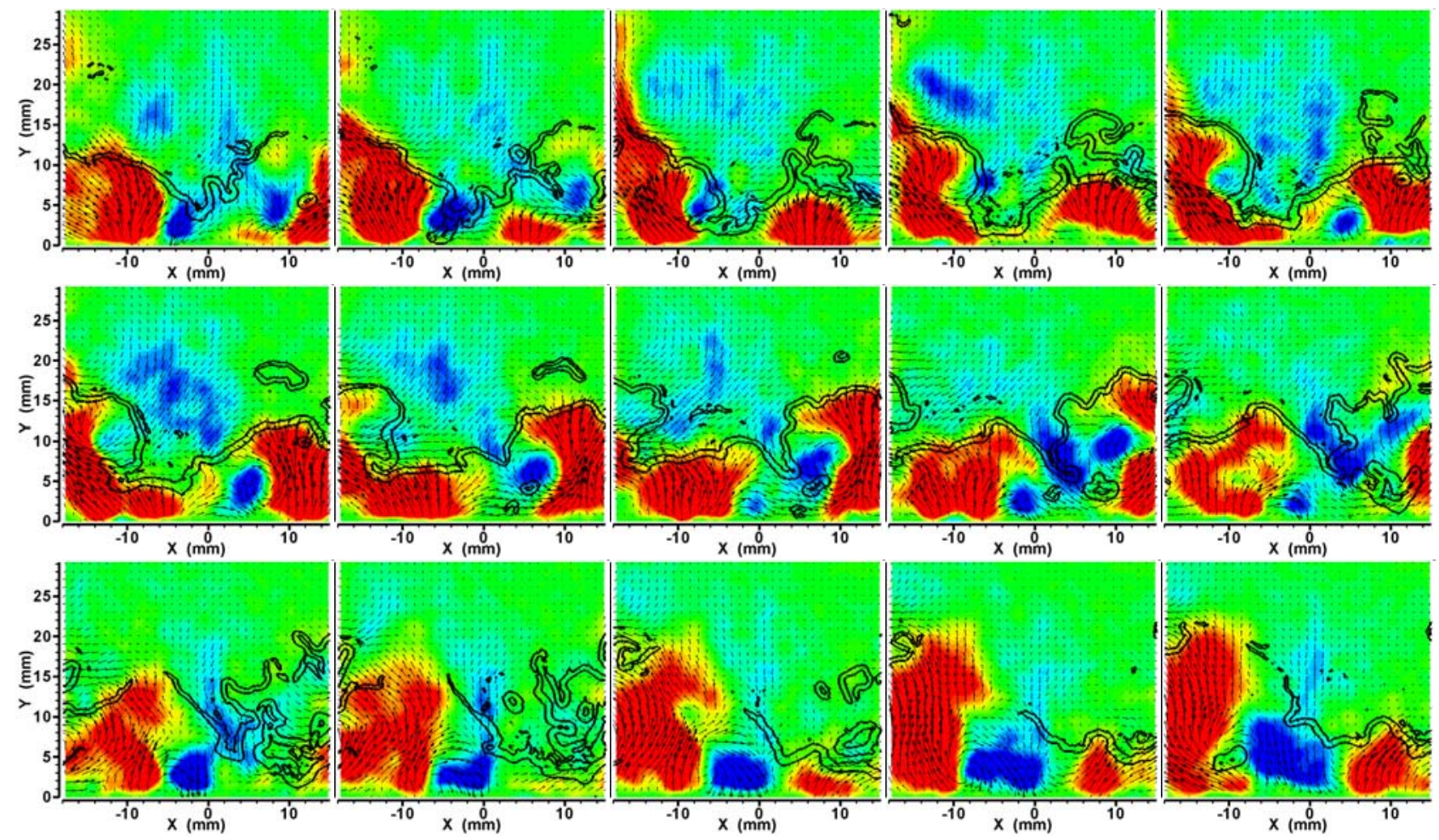

\section{$\begin{array}{lllllllllll}-7.5 & -6 & -4.5 & -3 & -1.5 & 0 & 1.5 & 3 & 4.5 & 6 & 7.5(\mathrm{~m} / \mathrm{s})\end{array}$}

Figure 8. c) Reaction zones overlaid against contours of axial velocity. For clarity, only every second computed vector is plotted in the vector overlay. 

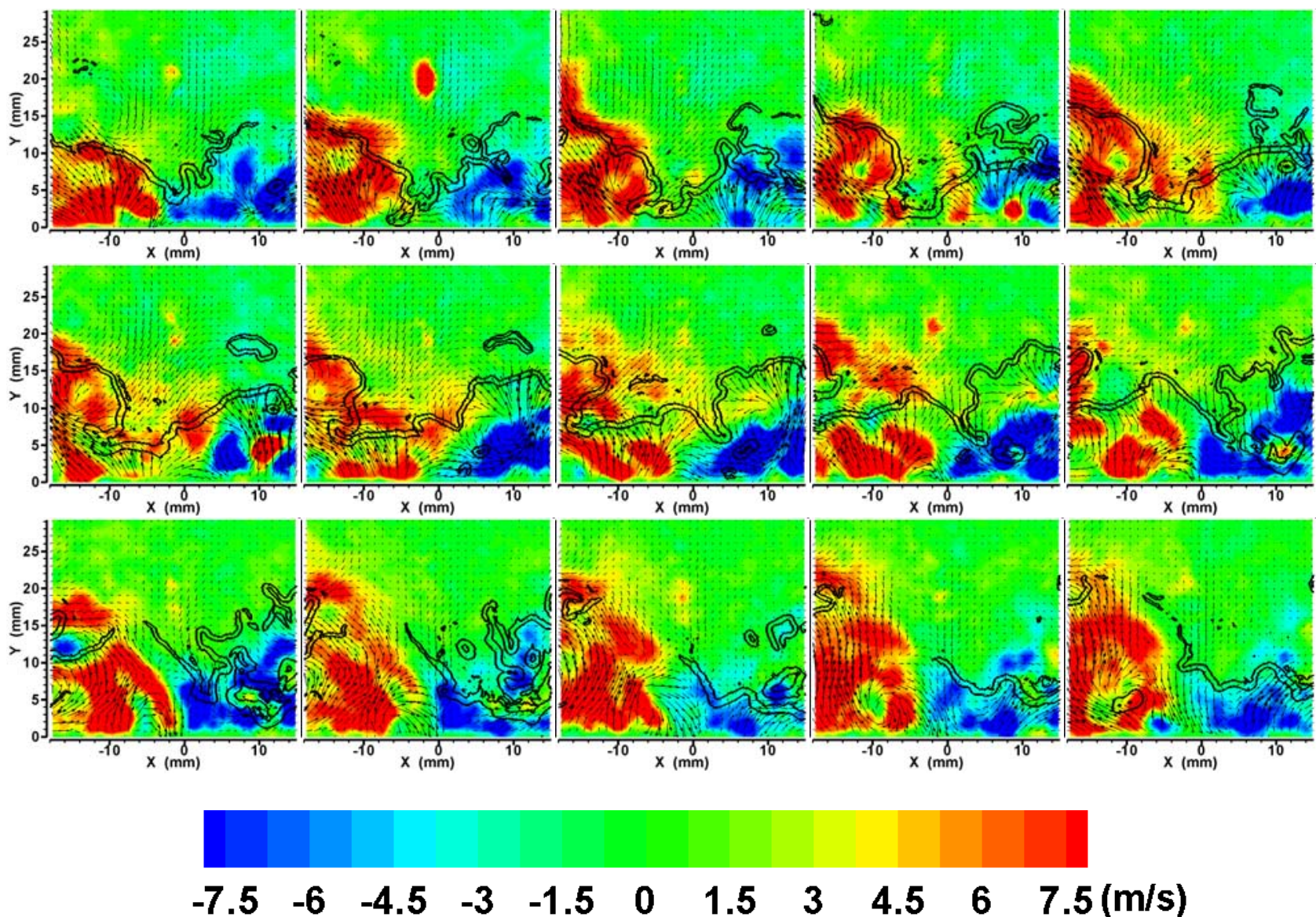

Figure 8. d) Reaction zones overlaid against contours of Vz- (through plane) velocity. For clarity, only every second computed vector is plotted in the vector overlay. 

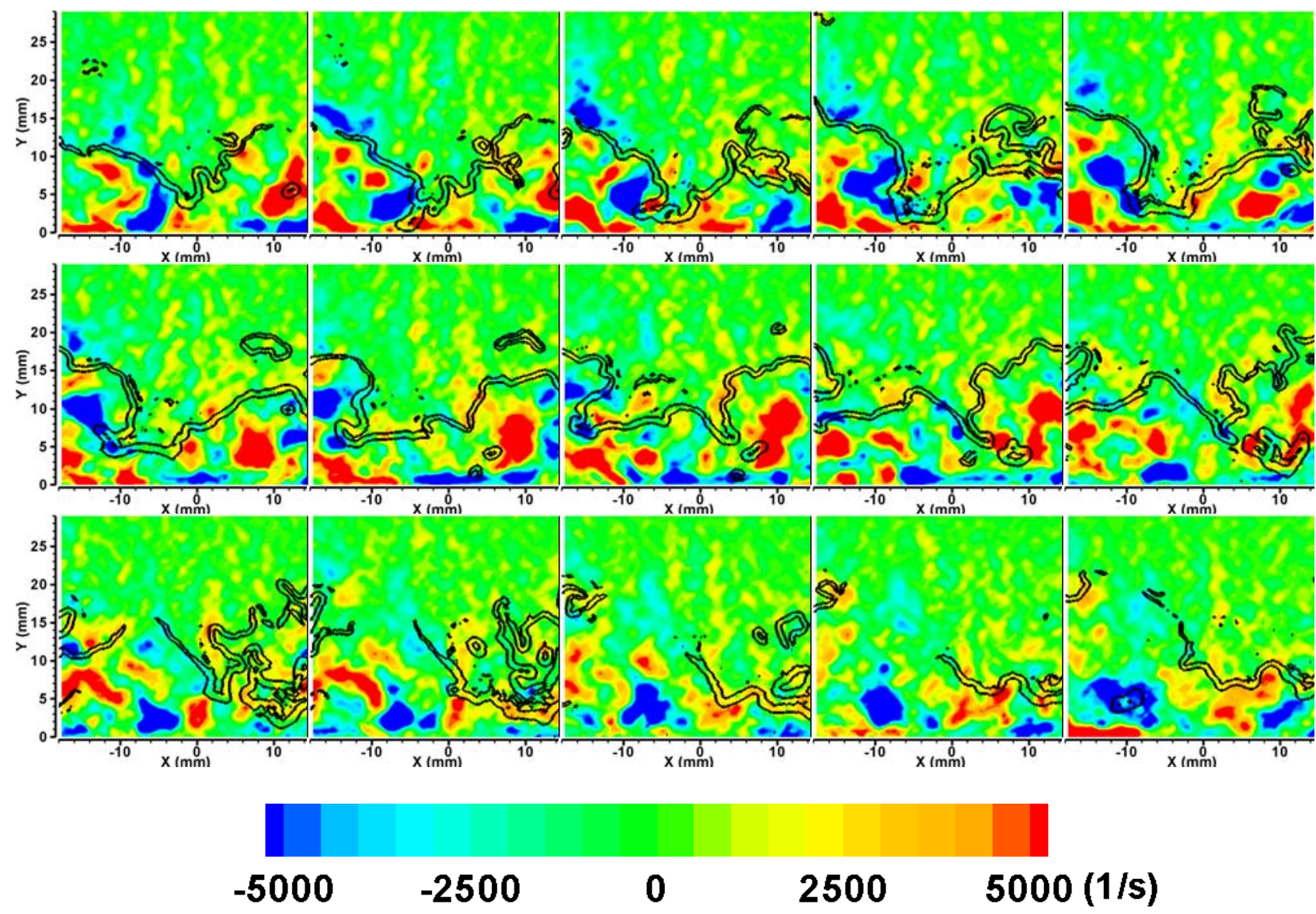

Figure 8. e) Reaction zones overlaid against contours of vorticity 

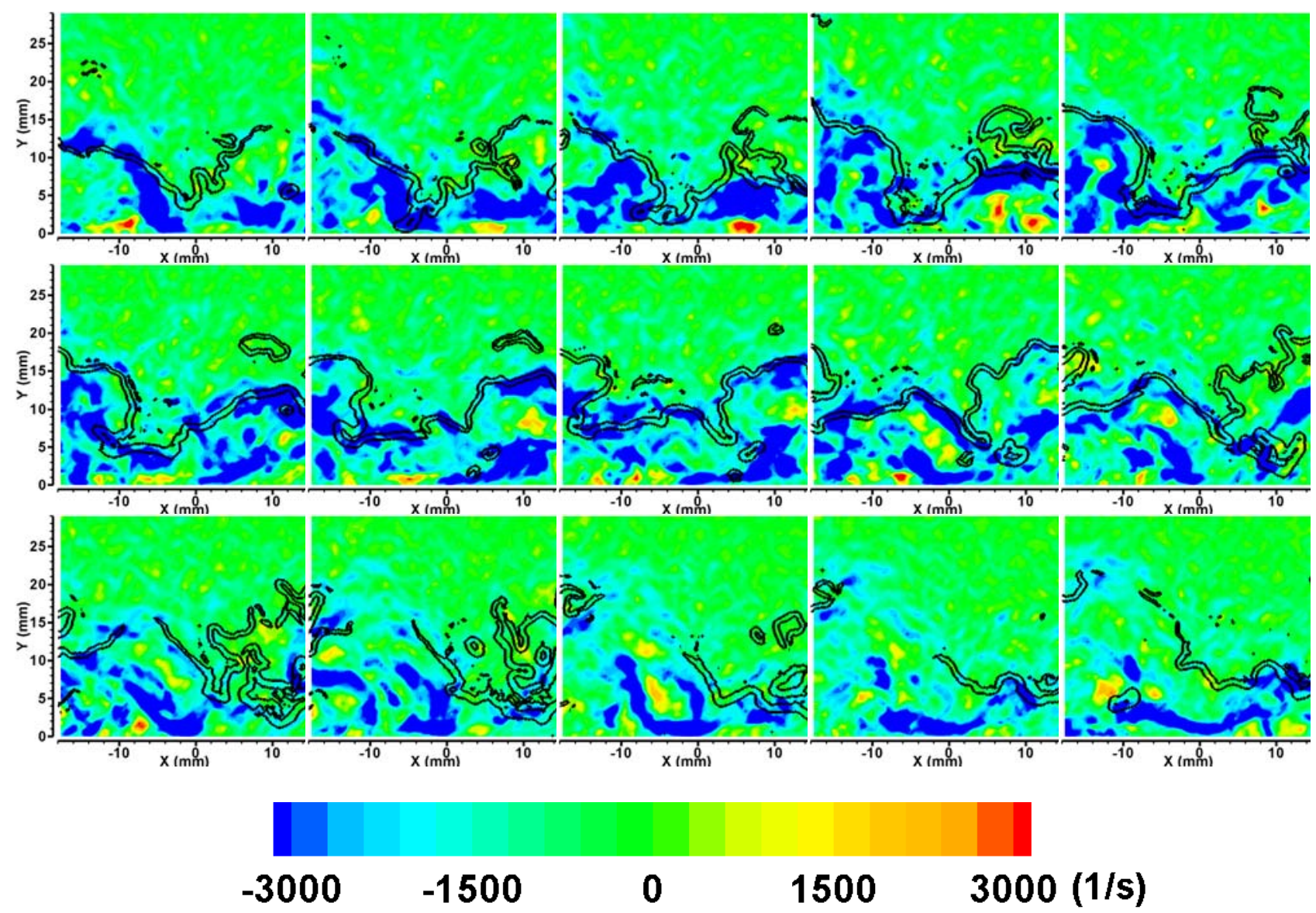

Figure 8. f) Reaction zones overlaid against contours of minimum principle normal strain 


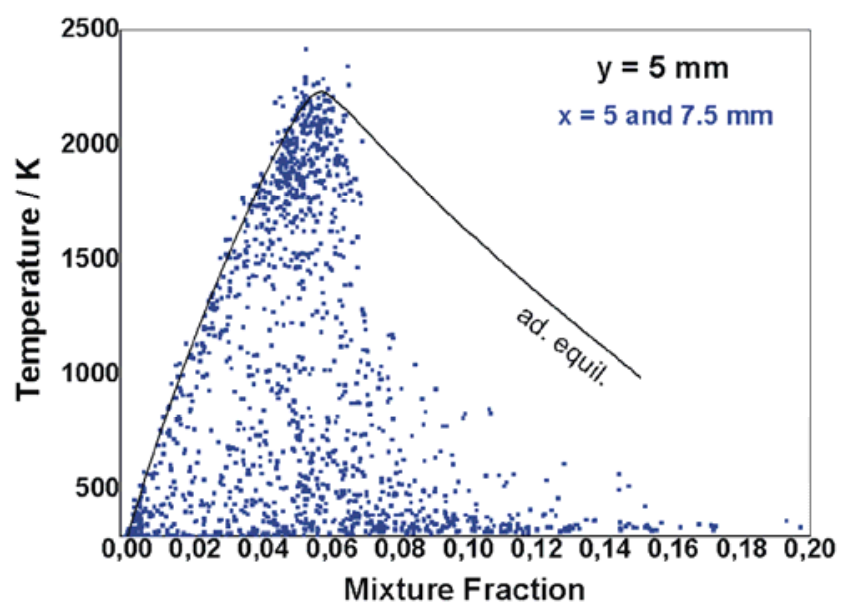

Figure 9. Scatter plot of data acquired by Meier et al. [2006] showing evidence of auto-ignition around location where isolated $\mathrm{OH}$ pockets appear in the image sequences. 

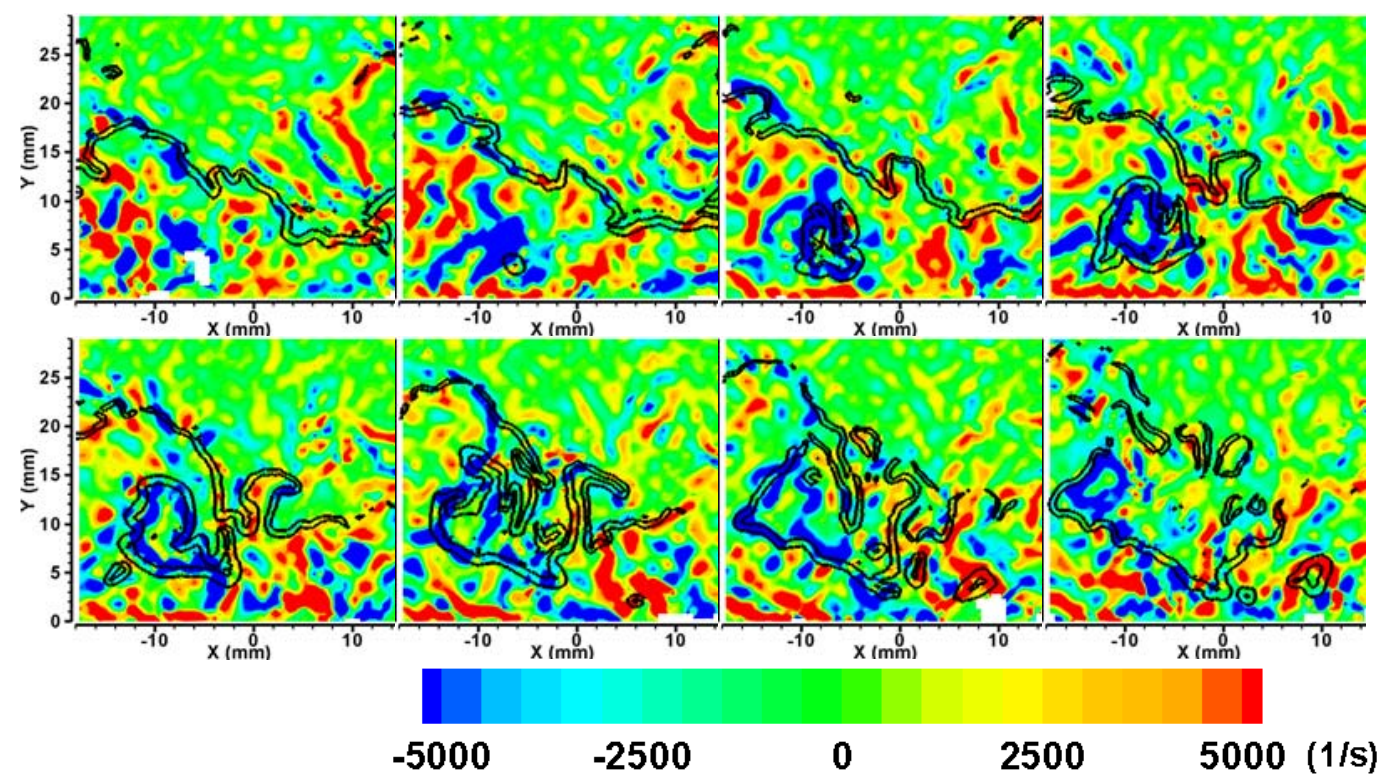

Figure 10. Development of isolated $\mathrm{OH}$ pocket (first evident near $\mathrm{x}=-5 \mathrm{~mm}$ in frame 2), overlaid on unfiltered vorticity data. 